\title{
FÓRMULAS DE NUTRIÇÃO ENTÉRICA E SUA APLICABILIDADE EM IDADE PEDIÁTRICA
}

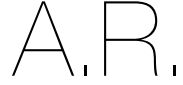
ARTIGO DE REVISÃO

1 Unidade de Nutrição Pediátrica - Centro Materno Infantil do Centro Hospitalar Universitário São João,

Alameda Prof. Hernâni

Monteiro,

4200-319 Porto, Portugal

2 Serviço de Nutrição do Centro Hospitalar Universitário São João, Alameda Prof. Hernâni

Monteiro,

4200-319 Porto, Portugal

${ }^{3}$ Faculdade de Ciências de Nutrição e Alimentação da Universidade do Porto,

Rua do Campo Alegre,

n. ${ }^{\circ} 823$,

4150-180 Porto, Portugal

${ }^{4}$ Center for Health

Techonology and Services Research,

Rua Dr. Plácido da Costa,

4200-450 Porto, Portugal

${ }^{5}$ Cooperativa de Ensino Superior Politécnico e

Universitário,

Rua Central de Gandra,

n. ${ }^{\circ} 1317$

4585-116 Gandra

Portugal

${ }^{6}$ Faculdade de Medicina da Universidade Porto, Rua Dr. Plácido da Costa, $\mathrm{s} / \mathrm{n}$,

4200-450 Porto, Portugal

Endereço para correspondência:

Diana e Silva

Centro Hospitalar Universitário

de São João - Serviço de

Nutrição,

lameda Prof. Hernâni Monteiro,

200-319 Porto, Portugal

diana.mv.silva@gmail.com

Histórico do artigo:

Recebido a 17 de abril de 202 Aceite a 21 de setembro de 2021

\section{ENTERAL NUTRITION FORMULAS AND THEIR APPLICABILITY IN PEDIATRIC AGE}

Diana e Silva ${ }^{1-4 *}$; Marta Rola²; Ana Barreira Lopes ${ }^{3} ;$ Maria do Céu Monteiro 3,5 ; Paula Guerra'; António Guerra33,4,

RESUMO

A utilização de nutrição entérica tem um papel importante na recuperação da criança ou adolescente desnutrido, ou em risco de desnutrição. Atualmente, existe uma ampla gama de dietas entéricas que surgiram ao longo dos últimos 15 anos, como consequência do avanço tecnológico e do conhecimento relativo às necessidades em macro e micronutrientes adequadas a cada doente. É por isso objetivo do presente trabalho fornecer uma atualização qualitativa, e quantitativa, das fórmulas entéricas que se encontram disponíveis no mercado português até ao mês de janeiro de 2021

\section{PALAVRAS-CHAVE}

Dietas entéricas, Legislação, Nutrição, Nutrição entérica, Pediatria, Suplementação nutricional

ABSTRACT

The use of enteral nutrition plays an important role in the recovery of the malnourished child or adolescent, or at risk of malnutrition. Currently, there is a wide range of enteral diets that have appeared in the Portuguese market over the last 15 years, a consequence of the importance to meet the different nutritional needs of each patient. It is therefore the objective of this article to provide a qualitative and quantitative update of the enteral formulas available in Portuguese until January 2021

KEYWORDS

Enteral diets, Legislation, Nutrition, Enteral nutrition, Pediatrics, Nutritional supplementation

\section{INTRODUÇÃO}

Este artigo tem como objetivo principal proceder à descrição e atualização das fórmulas entéricas (alimentos para fins medicinais específicos) disponíveis relativamente à anterior publicação há cerca de 15 anos (1). Simultaneamente pretende-se que desta forma, seja mais fácil e clara a escolha deste tipo de dietas, tendo em vista as suas caraterísticas nutricionais e os fins a que se destinam.

\section{Alimentos para Fins Medicinais Específicos}

\section{Legislação}

A Diretiva n. ${ }^{0}$ 1999/21/CE (2) veio fixar normas específicas aplicáveis a alimentos destinados a alimentação especial e constituiu a primeira legislação que regulou a composição em micronutrientes das dietas entéricas e dos suplementos administrados por sonda ou via oral. Presentemente, os alimentos para fins medicinais específicos encontram-se normalizados na UE pelo Regulamento (UE) n. ${ }^{\circ}$ 609/2013, relativo aos alimentos para grupos específicos (3) e pelo Regulamento Delegado UE 2016/128 (4). O Regulamento (UE) n. ${ }^{\circ}$ 609/2013 estabelece requisitos em matéria de composição e informação para certas categorias de alimentos, estabelece uma lista da UE de substâncias que podem ser adicionadas a certas categorias de alimentos e define as regras para a aprovação e atualização dessa lista. $O$ Regulamento Delegado (UE) 2016/128 (4) revoga e substitui, a partir de 22 de fevereiro de 2019, a Diretiva 1999/21/CE, que estabelecia requisitos espećficos para os alimentos para fins medicinais específicos nos termos da Diretiva 2009/39/CE (5). A legislação em vigor considera as regras básicas relativamente ao teor em vitaminas e substâncias minerais em alimentos destinados a fins medicinais específicos definidas na Diretiva 1999/21/CE, dado que até agora garantiram um quadro adequado para estes alimentos. As regras incluem teores mínimos e máximos, no caso de produtos considerados nutricionalmente completos para satisfazer as necessidades nutricionais do doente $e$ apenas teores máximos no caso de produtos considerados nutricionalmente incompletos. Estes valores são expressos por $100 \mathrm{kcal}$, não fazendo referência ao teor de proteínas, dos glícidos ou dos lípidos. Estas diretivas consideram não existirem diferenças relativas às necessidades de vitaminas e minerais em crianças com idade superior a um ano e os adultos, e os valores mínimos e máximos 
têm por base o consumo médio diário de 2000kcal de um adulto. O cálculo para as necessidades nutricionais na criança poderá basearse nos valores de referência das Recomendações Dietéticas da Food and Nutrition Board - Institute of Medicine (6-12).

As substâncias que podem ser adicionadas aos alimentos para fins medicinais específicos e que constam da Lista da UE (3) incluem: 1) vitaminas; 2) sais minerais; 3) aminoácidos; 4) carnitina e taurina; 5) nucleótidos; e 6) colina e inositol. A legislação em vigor considera que, dada a ampla diversidade dos produtos e também a rápida evolução dos conhecimentos científicos nos quais se baseiam, não é conveniente estabelecer normas detalhadas em matéria de composição. No entanto, qualquer modificação inovadora com base no progresso científico e técnico deve ser decidida em conformidade com o processo previsto nas respetivas diretivas.

\section{Definições e Designações}

Os alimentos para Fins Medicinais Específicos são definidos no Regulamento (UE) n. ${ }^{\circ}$ 609/2013 (3), como «alimentos especialmente transformados ou compostos e destinados a satisfazer os requisitos nutricionais de pacientes, incluindo lactentes e para consumo sob supervisão médica. Destinam-se à alimentação exclusiva ou parcial de pacientes com capacidade limitada, diminuída ou alterada para ingerir, digerir, absorver, metabolizar ou excretar alimentos correntes ou alguns dos nutrientes neles contidos ou metabolitos, ou cujo estado de saúde determine requisitos nutricionais particulares que não possam ser satisfeitos por uma modificação do regime alimentar normal». De acordo com o Regulamento Delegado (UE) 2016/128, estes alimentos são «classificados nas três categorias seguintes:

1. Produtos alimentares nutricionalmente completos, com fórmula dietética padrão, os quais, consumidos em conformidade com as instruções do fabricante, podem constituir a única fonte alimentar para as pessoas a que se destinam;

2. Produtos alimentares nutricionalmente completos, com fórmula dietética adaptada a doenças, distúrbios ou problemas de saúde específicos, os quais, consumidos em conformidade com as instruções do fabricante, podem constituir a única fonte alimentar para as pessoas a que se destinam;

3. Produtos alimentares nutricionalmente incompletos, com fórmula padrão ou fórmula dietética adaptada a doenças, distúrbios ou problemas de saúde específicos, não adequados a uma utilização como fonte alimentar única».

Os produtos alimentares referidos nos pontos 1 e 2 podem também ser utilizados como substituto parcial ou suplemento da dieta do doente. Os alimentos para fins medicinais específicos são ainda classificados em função da sua composição em macronutrientes em dietas poliméricas, semielementares ou oligoméricas e em elementares ou monoméricas e que abaixo se descriminam.

\section{Classificação das Dietas Entéricas}

Os produtos de nutrição entérica, existentes no mercado, são na sua maioria produtos sob a forma líquida, preparados para serem consumidos no momento. Contudo, também existem alguns produtos, sob a forma de pó, que devem ser preparados, recorrendo a água, leite ou fórmula infantil como solventes (13).

A composição nutricional das fórmulas entéricas deve ser adaptada à idade da criança e do adolescente. Como anteriormente referido, e apesar de não existir evidência científica, o Comité de Nutrição da European Society for Paediatric Gastroenterology, Hepatology and Nutrition (ESPGHAN) refere que na ausência de produtos de nutrição entérica para a idade pediátrica, a partir dos 8-10 anos de idade, poderão ser usados produtos indicados para adultos (13).
Nesse sentido, o presente artigo irá referir os produtos destinados à idade pediátrica, bem como os destinados a adultos, ressalvando que estes últimos só poderão ser usados a partir dos 8-10 anos de idade.

\section{Em Função da Composição Estrutural dos Macro-nutrientes}

\section{a) Fórmulas Poliméricas}

As fórmulas poliméricas são constituídas por nutrientes na sua forma intacta e são nutricionalmente completas. Como tal, requerem um sistema digestivo funcional, com normal capacidade de digestão e absorção. Estas fórmulas são isentas de lactose e na sua maioria também de glúten (14).

Relativamente à sua composição nutricional, contêm proteínas inteiras; hidratos de carbono provenientes de oligossacarídeos, maltodextrinas ou amido; lípidos derivados de óleos vegetais; minerais; vitaminas e oligoelementos (14)

Os hidratos de carbono são a principal fonte de energia, devendo fornecer cerca de 40 a $60 \%$ de energia diariamente. A principal fonte de hidratos de carbono nestas fórmulas são as maltodextrinas. As maltodextrinas são mais solúveis que o amido, apresentando menor carga osmótica. Algumas fórmulas podem conter também amido ou sacarose, em pequena quantidade, o que melhora a palatabilidade da fórmula.

As fibras correspondem a hidratos de carbono não digeríveis no intestino delgado, e que chegam ao cólon de forma total ou parcialmente disponíveis em termos metabólicos e energéticos (14). As fibras e os produtos produzidos na sua fermentação, os ácidos gordos de cadeia curta, apresentam potenciais efeitos benéficos na fisiologia intestinal e na prevenção de diarreia e obstipação $(15,16)$, bem como na absorção de nutrientes, no metabolismo de hidratos de carbono e lípidos, entre outros (14). Algumas fórmulas poliméricas podem conter fibras na sua composição, contudo as vantagens da sua inclusão continuam a ser controversas (14).

As proteínas fornecem entre 15 a 25\% do total de energia nas fórmulas poliméricas. A proteína presente neste tipo de fórmulas pode estar na sua origem natural (exemplo: leite, ovo) ou sob a forma de isolado de proteína (exemplo: caseína, proteína do soro, isolado de proteína de soja, clara de ovo, albumina de ovo) (14).

Por sua vez, os lípidos fornecem 25 a 40\% do total de energia presente neste tipo de fórmulas. Os lípidos têm uma densidade calórica superior, contribuindo significativamente para o conteúdo energético não-proteico das fórmulas poliméricas. As fontes de lípidos mais usadas neste tipo de fórmulas são o óleo de milho e de soja. Estes óleos têm como característica comum a presença de triglicerídeos de cadeia longa, ajudando a limitar a osmolalidade (14).

Algumas fórmulas poliméricas podem conter triglicerídeos de cadeia média (TCM), constituindo parte ou a totalidade do conteúdo lipídico. A vantagem da utilização de TCM é o da sua absorção facilitada, pois são assimilados diretamente para a circulação portal, contornando a circulação linfática e não necessitando de sais biliares, nem da lípase pancreática. Como tal, a utilização de TCM pode ser útil em casos de malabsorção ou de quilotoráx. Contudo, é importante salientar que os TCM não contêm os ácidos gordos essenciais (14).

Normalmente, as fórmulas poliméricas fornecem $100 \%$ das necessidades em vitaminas, minerais e oligoelementos. Em casos especiais, quando as necessidades se encontram aumentadas ou ocorre perda de nutrientes, é importante avaliar a necessidade da sua suplementação (14).

A osmolaridade destas fórmulas aproxima-se dos níveis fisiológicos (333mOsml/L), constituindo uma vantagem relativamente à sua tolerância (14).

Discriminam-se nas Tabelas 1 e 2 as dietas poliméricas e respetivas composições em macro e micronutrientes. 
Dietas poliméricas hiperenergéticas disponíveis no mercado português

\begin{tabular}{|c|c|c|c|c|c|c|c|c|c|c|c|c|c|c|c|c|}
\hline \multicolumn{17}{|c|}{ DIETAS POLIMÉRICAS HIPERENERGÉTICAS } \\
\hline $\begin{array}{l}\text { DESIGNACCÃO } \\
\text { COMERCIAL } \\
\end{array}$ & A1 & A2 & A3 & A4 & A5 & A6 & A7 & A8 & A9 & A10 & A11 & A12 & A13 & A14 & A15 & A16 \\
\hline $\begin{array}{c}\text { CARACTERÍSTICAS } \\
\text { NUTRICIONAIS/100ML } \\
\text { OU } 100 \mathrm{G}\end{array}$ & $100 \mathrm{ML}$ & $100 \mathrm{ML}$ & $100 \mathrm{ML}$ & $100 \mathrm{ML}$ & $100 \mathrm{ML}$ & $100 \mathrm{ML}$ & $100 \mathrm{ML}$ & $\begin{array}{l}\text { POR } 100 \mathrm{ML} \\
\text { (preparacáo standard: } 1 \\
\text { saqueta de pó }+240 \mathrm{ml} \\
\text { de leite gordo) }\end{array}$ & $100 \mathrm{ML}$ & $100 \mathrm{ML}$ & $100 \mathrm{G}$ & $100 \mathrm{ML}$ & $100 \mathrm{ML}$ & $100 \mathrm{ML}$ & $100 \mathrm{ML}$ & $100 \mathrm{ML}$ \\
\hline ENERGIA (kcal/ kJ) & $150 / 630$ & $150 / 630$ & $151 / 635$ & $152 / 640$ & $150 / 630$ & $150 / 630$ & $150 / 630$ & $500 / 2100$ & $200 / 840$ & $200 / 840$ & $200 / 840$ & $320 / 1344$ & $150 / 630$ & $150 / 630$ & $200 / 840$ & $200 / 840$ \\
\hline PROTEÍNAS (g) & 3,8 & 3,8 & 4 & 4,8 & 5,6 & 4 & 10 & 4,1 & 10 & 10 & 10 & 16 & 5,6 & 7,5 & 10 & 10 \\
\hline LíPIDOS (g) & 1,3 & 1,3 & & & 0 & 0 & 0 & 0 & 0 & 0 & 0 & 0 & 0 & 3,3 & 2,6 & 2,6 \\
\hline $\mathrm{MCT}(\mathrm{g})$ & 0,4 & 0,5 & 0,8 & 0,8 & 0,4 & 0 & 0,6 & 16,7 & 0,6 & 0,7 & 0,6 & 1,1 & 0,5 & 0,4 & 0,6 & 0,6 \\
\hline AGS (g) & 3,3 & 3,2 & 3,9 & 3,7 & 3,8 & 0 & 4,9 & 6,2 & 5,8 & 5,8 & 5,8 & 9,9 & 3,7 & 0,5 & 5,2 & 5,2 \\
\hline AGMI (g) & 1,7 & 1,6 & 2,0 & 1,9 & 1,6 & 0 & 1,2 & 1,4 & 1,4 & 1,4 & 1,4 & 5 & 1,6 & 1,5 & 1,6 & 1,6 \\
\hline AGPI (g) & 18,7 & 18,1 & 18,5 & 18,5 & 18,8 & 33,5 & 12,1 & 65,5 & 22,5 & 21,7 & 22,5 & 28 & 18,8 & 17 & 17,5 & 16,7 \\
\hline $\begin{array}{l}\text { HIDRATOS DE } \\
\text { CARBONO (g) }\end{array}$ & 3,8 & 3,8 & 4 & 4,8 & 5,6 & 4 & 10 & 4,1 & 10 & 10 & 10 & 16 & 5,6 & 7,5 & 10 & 10 \\
\hline Lactose (g) & $\leq 0,05$ & $\leq 0,05$ & $<0,025$ & $<0,025$ & $\leq 0,27$ & $\leq 0,02$ & $\leq 0,4$ & 2,24 & $\leq 0,3$ & $\leq 0,3$ & 0,32 & $\leq 0,24$ & $\leq 0,1$ & $\leq 0,06$ & $\leq 0,25$ & $\leq 0,25$ \\
\hline $\begin{array}{l}\text { FIBRA SOLÚVEL/ } \\
\text { INSOLÚVEL (g) }\end{array}$ & 0 & 1,1 & 0,8 & 1,1 & & & & $<0,5$ & 0 & 1,6 & 0 & 0,5 & 0,04 & 0 & 0 & 1,5 \\
\hline \multicolumn{17}{|l|}{$\begin{array}{l}\text { MINERAIS/ } \\
\text { OLIGOELEMENTOS }\end{array}$} \\
\hline $\mathrm{Na}(\mathrm{mg})$ & 81 & 102 & 90 & 107 & 80 & 6 & 60 & 180 & 60 & 60 & 65 & 112 & 100 & 120 & 60 & 60 \\
\hline $\mathrm{K}(\mathrm{mg})$ & 150 & 150 & 165 & 186 & 135 & 7 & 135 & 94 & 160 & 160 & 167 & 312 & 207 & 234 & 170 & 170 \\
\hline $\mathrm{Cl}$ (mg) & 123 & 110 & 143 & 147 & 100 & 190 & 58 & & 80 & 60 & 62 & 144 & 153 & 184 & 90 & 90 \\
\hline $\mathrm{Ca}$ (mg) & 105 & 98 & 90 & 95 & 135 & 50 & 205 & 87 & 205 & 205 & 196 & 160 & 80 & 80 & 205 & 205 \\
\hline $\mathrm{P}(\mathrm{mg})$ & 92 & 97 & 75 & 90 & 80 & 11 & 120 & 58 & 120 & 120 & 125 & 112 & 63 & 63 & 120 & 120 \\
\hline $\mathrm{Mg}(\mathrm{mg})$ & 19 & 20 & 17 & 26 & 21 & 1 & 18 & 8 & 16 & 16 & 16 & 40 & 27 & 27 & 20 & 20 \\
\hline $\mathrm{Fe}(\mathrm{mg})$ & 1,5 & 1,5 & 1,5 & 2,0 & 2 & 2,5 & 2,5 & & 2,5 & 2,5 & 2,5 & 4,8 & 1,33 & 1,33 & 2,67 & 2,67 \\
\hline $\mathrm{Zn}$ (mg) & 1,5 & 1,5 & 1,5 & 1,7 & 1,5 & 1,88 & 2 & & 1,6 & 1,6 & 2 & 3,5 & 1,2 & 1,2 & 2,4 & 2,4 \\
\hline I ( $\mu \mathrm{g})$ & 15 & 15 & 15 & 17 & 30 & 37,5 & 37,5 & & 37,5 & 37,5 & 37,6 & 44,8 & 13,3 & 13,3 & 26,7 & 26,7 \\
\hline $\mathrm{Cu}(\mu \mathrm{g})$ & 150 & 150 & 10 & 0,2 & 0,3 & 375 & 375 & & 375 & 375 & 375 & 512 & 130 & 133 & 267 & 267 \\
\hline $\mathrm{Mn}(\mathrm{mg})$ & 0,18 & 0,18 & 0,2 & 0,4 & 0,4 & 0,5 & 0,5 & & 0,5 & 0,5 & 0,5 & 1,2 & 0,27 & 0,27 & 0,53 & 0,53 \\
\hline $\mathrm{F}(\mathrm{mg})$ & 0,12 & 0,12 & 0,1 & 0,1 & 0,2 & 0,25 & 0,25 & & 0,25 & 0,25 & 0,25 & 0,4 & 0,13 & 0,13 & 0,27 & 0,27 \\
\hline $\mathrm{Se}(\mu \mathrm{g})$ & 4,5 & 4,5 & 4,5 & 7,4 & 10 & 12,5 & 13,5 & & 13,5 & 13,5 & 13,5 & 23 & 6,7 & 6,7 & 13,3 & 13,3 \\
\hline $\mathrm{Cr}(\mu \mathrm{g})$ & 6 & 9 & 5,3 & 7,7 & 10 & 12,5 & 12,5 & & 12,5 & 12,5 & 13 & 23 & 6,7 & 6,7 & 13,3 & 13,3 \\
\hline Mo $(\mu \mathrm{g})$ & 6 & 6 & 6,0 & 11 & 15 & 18,8 & 18,8 & & 18,75 & 18,75 & 18,8 & 30,4 & 10 & 10 & 20 & 20 \\
\hline \multicolumn{17}{|l|}{ VITAMINAS } \\
\hline Vitamina A ( $\mu \mathrm{g})(\mathrm{RE})$ & 68 & 68 & 61 & 92 & 120 & 150 & 0,15 & & 150 & 150 & 150 & 224 & 70 & 70 & 140 & 140 \\
\hline Vitamina D $(\mu \mathrm{g})$ & 1,13 & 1,13 & 1,5 & 1,1 & 2 & 2,5 & 2,5 & & 5 & 5 & 5 & 8 & 1,3 & 1,33 & 2 & 2 \\
\hline Vitamina E (mg) (a-TE) & 3 & 3 & 1,9 & 1,9 & 3 & 3,75 & 3,75 & & 3,75 & 3,75 & 3,75 & 5,76 & 3 & 1,33 & 2,67 & 2,67 \\
\hline Vitamina K ( $\mu \mathrm{g})$ & 6 & 6 & 6,0 & 6,8 & 16,7 & 25 & 21 & & 21 & 21 & 21 & 20,8 & 6,67 & 6,67 & 13,3 & 13,3 \\
\hline Tiamina (mg) & 0,3 & 0,3 & 0,2 & 0,2 & 0,23 & 0,3 & 0,3 & & 0,3 & 0,3 & 0,3 & 0,5 & 0,13 & 0,13 & 0,27 & 0,27 \\
\hline Riboflavina (B2) (mg) & 0,3 & 0,3 & 0,2 & 0,2 & 0,32 & 0,4 & 0,4 & & 0,4 & 0,4 & 0,4 & 0,51 & 0,17 & 0,17 & 0,35 & 0,35 \\
\hline Niacina (mg) (NE) & 1,8 & 1,8 & 1,7 & 2,3 & 3 & 3,75 & 3,75 & & 5,58 & 5,58 & 3,75 & 5,4 & 1,6 & 1,6 & 3,2 & 3,2 \\
\hline Ácido Pantoténico (mg) & 0,6 & 0,6 & 0,5 & 0,7 & 1,2 & 1,5 & 1,5 & & 1,5 & 1,5 & 1,5 & 1,92 & 0,47 & 0,47 & 0,93 & 0,93 \\
\hline Vitamina B6 (mg) & 0,17 & 0,17 & 0,2 & 0,2 & 0,33 & 0,43 & 0,43 & & 0,43 & 0,43 & 0,43 & 0,58 & 0,16 & 0,16 & 0,32 & 0,32 \\
\hline Ácido Fólico ( $\mu \mathrm{g})$ & 33 & 33 & 23 & 32 & 50 & 62,5 & 62,5 & & 62,5 & 62,5 & 62,5 & 67,2 & 26,7 & 27 & 53,3 & 53,3 \\
\hline Vitamina B12 $(\mu \mathrm{g})$ & 0,3 & 0,3 & 0,3 & 0,3 & 0,6 & 0,75 & 0,75 & & 0,75 & 0,75 & 0,75 & 1,1 & 0,27 & 0,27 & 0,53 & 0,53 \\
\hline Biotina $(\mu \mathrm{g})$ & 7,5 & 7,5 & 6,0 & 6,0 & 7,5 & 9,4 & 9,4 & & 9,4 & 9,4 & 9,4 & 14,4 & 5 & 5 & 10 & 10 \\
\hline Vitamina C (mg) & 12 & 12 & 15 & 15 & 15 & 18,8 & 18,8 & & 18,8 & 18,8 & 18,8 & 41,6 & 6,67 & 6,7 & 13,3 & 13,3 \\
\hline \multicolumn{17}{|l|}{$\begin{array}{l}\text { DISTRIBUIÇÃO DE } \\
\text { ENERGIA }\end{array}$} \\
\hline Proteína (En \%) & 10,2 & 10,2 & 10,7 & 12,6 & 15 & 11 & 27 & 3 & 20 & 20 & 20 & 20 & 15 & 20 & 20 & 20 \\
\hline Lípidos (En \%) & 40 & 40 & 39,6 & 37,5 & 35 & 0 & 40 & 44 & 35 & 35 & 35 & 45 & 35 & 35 & 45 & 45 \\
\hline $\begin{array}{l}\text { Hidratos de } \\
\text { Carbono (En \%) }\end{array}$ & 49,8 & 48,3 & 48,7 & 48,4 & 50 & 89 & 32,3 & 53 & 45 & 43,4 & 45 & 34,7 & 50 & 45 & 35 & 35 \\
\hline $\begin{array}{l}\text { OSMOLARIDADE } \\
\text { (mOsml/L) }\end{array}$ & 400 & 420 & 315 & 315 & 355 & 680 & 390 & 900 & 590 & 590 & $\mathrm{n}, \mathrm{a}$ & $630 / 697$ & 330 & 300 & 395 & 395 \\
\hline SABORES & Morango & Chocolate & Neutro & Neutro & $\begin{array}{l}\text { Baunilha, } \\
\text { Cappucci- } \\
\text { no, Frutos } \\
\text { Tropicais, } \\
\text { Morango }\end{array}$ & $\begin{array}{l}\text { Ananás, } \\
\text { Maçã }\end{array}$ & $\begin{array}{l}\text { Baunilha, } \\
\text { Chocolate, } \\
\text { Morango } \\
\text { Silvestre }\end{array}$ & $\begin{array}{l}\text { Banana, Baunilha, } \\
\text { Chocolate, } \\
\text { Morango }\end{array}$ & $\begin{array}{l}\text { Baunilha, } \\
\text { Frutos } \\
\text { Silvestres }\end{array}$ & Chocolate & $\begin{array}{c}\text { Baunilha, } \\
\text { Cappuc- } \\
\text { cino, } \\
\text { Chocolate, } \\
\text { Morango } \\
\text { Silvestre, } \\
\text { Praliné }\end{array}$ & $\begin{array}{l}\text { Baunilha- } \\
\text {-caramelo, } \\
\text { Avelã }\end{array}$ & Neutro & Neutro & Neutro & Neutro \\
\hline VIA ADMINISTRAÇÃO & Oral & Oral & Sonda & Sonda & Oral & Oral & Oral & Oral & Oral & Oral & Oral & Oral & Sonda & Sonda & Sonda & Sonda \\
\hline
\end{tabular}

Produtos a sombreado correspondem a produtos específicos para a idade pediátrica 
Continuação

\begin{tabular}{|c|c|c|c|c|c|c|c|c|c|c|c|c|c|c|c|c|}
\hline & & & & & & DIETAS POL & LIMÉRICAS H & HIPERENERC & QEÉTICAS & & & & & & & \\
\hline $\begin{array}{l}\text { DESIGNACCÃO } \\
\text { COMERCIAL } \\
\end{array}$ & A17 & A18 & A19 & A20 & A21 & A22 & A23 & A24 & A25 & A26 & A27 & A28 & A29 & A30 & A31 & A32 \\
\hline $\begin{array}{c}\text { CARACTERISTICAS } \\
\text { NUTRICIONAIS/100ML } \\
\text { OU } 100 \mathrm{G}\end{array}$ & $100 \mathrm{G}$ & $100 \mathrm{ML}$ & $100 \mathrm{ML}$ & & $100 \mathrm{ML}$ & $100 \mathrm{ML}$ & $100 \mathrm{G}$ & $100 \mathrm{ML}$ & $100 \mathrm{ML}$ & $100 \mathrm{ML}$ & $100 \mathrm{ML}$ & $100 \mathrm{ML}$ & $100 \mathrm{G}$ & $100 \mathrm{ML}$ & $100 \mathrm{G}$ & $100 \mathrm{ML}$ \\
\hline ENERGIA (kcal/ kJ) & $493 / 2065$ & $153 / 640$ & $151 / 637$ & $224,8 / 944$ & $160 / 670$ & $200 / 840$ & $170 / 692$ & $157 / 661$ & $240 / 1010$ & 245 / 1032 & $150 / 630$ & $240 / 1010$ & $161 / 675$ & $150 / 625$ & 435 / 1830 & $150 / 630$ \\
\hline PROTEÍNAS (g) & 11,1 & 3,3 & 5,6 & 14 & 10 & 9 & 10 & 6,1 & 14,4 & 14,6 & 18,0 & 9,6 & 9,9 & 5,9 & 21,8 & 6,0 \\
\hline LíPIDOS (g) & & & & 0 & & & & 1,24 & & & & & & & & \\
\hline MCT (g) & 5,7 & 0,7 & 0,7 & 0,752 & 0,9 & 0,7 & 1 & 2 & 0,9 & 0,86 & 0,6 & 0,9 & 0,7 & 0,6 & 3,4 & 1,5 \\
\hline AGS (g) & 13,6 & 4,1 & 1,9 & 5,504 & 3,6 & 5,7 & 4,3 & 2,8 & & & & & & & & 3,3 \\
\hline AGMI (g) & 3,3 & 2,0 & 2,3 & 2,16 & 1,7 & 2,3 & 2 & 1,4 & & & & & & & & 1,1 \\
\hline AGPI (g) & 61,4 & 18,8 & 21 & 22,4 & 16 & 21,4 & 16 & 19,3 & 24,4 & 25,3 & 16,7 & 29,7 & 19,0 & 18,4 & 53,4 & 18,3 \\
\hline $\begin{array}{l}\text { HIDRATOS DE } \\
\text { CARBONO (g) }\end{array}$ & 11,1 & 3,3 & 5,6 & 14 & 10 & 9 & 10 & 6,1 & 14,4 & 14,6 & 18,0 & 9,6 & 9,9 & 5,9 & 21,8 & 6,0 \\
\hline Lactose (g) & $<0,1$ & $<0,025$ & $<0,5$ & 0,8 & $<0,5$ & $<0,5$ & & & 0,3 & $<0,35$ & 2,1 & $<0,5$ & 0,4 & $<0,025$ & 0,9 & $<0,025$ \\
\hline $\begin{array}{l}\text { FIBRA SOLÚVEL/ } \\
\text { INSOLÚVEL (g) }\end{array}$ & 0 & 1,5 & 0 & 0 & 0 & 0 & $<0,2$ & 0 & & 0 & $<0,5$ & & $<0,5$ & & 2,6 & $<0,1$ \\
\hline $\begin{array}{l}\text { MINERAIS/ } \\
\text { OLIGOELEMENTOS }\end{array}$ & & & & 0 & & & & & & & & & & & & \\
\hline $\mathrm{Na}(\mathrm{mg})$ & 222 & 67 & 80 & 24,8 & 95 & 100 & & 120 & 40 & 40 & 60 & 96 & 80 & 90 & 314 & 134 \\
\hline $\mathrm{K}(\mathrm{mg})$ & 494 & 140 & 170 & 149,6 & 190 & 210 & & 190 & 105 & 97,6 & 208 & 236 & 215 & 159 & 693 & 201 \\
\hline $\mathrm{Cl}(\mathrm{mg})$ & 334 & 100 & 185 & 55,2 & 125 & 110 & & 105 & 60 & 60 & 83 & 91 & 125 & 87 & 392 & 100 \\
\hline $\mathrm{Ca}(\mathrm{mg})$ & 277 & 84 & 80 & 240 & 125 & 175 & 120 & 110 & 350 & 350 & 216 & 174 & 200 & 91 & 519 & 108 \\
\hline $\mathrm{P}(\mathrm{mg})$ & 244 & 75 & 80 & 149,6 & 125 & 120 & 105 & 90 & 300 & 282 & 165 & 174 & 200 & 78 & 281 & 108 \\
\hline $\mathrm{Mg}(\mathrm{mg})$ & 51 & 17 & 28 & 24,8 & 25 & 20 & & 18 & 55 & 54 & 31 & 33 & 36 & 23 & 74 & 34 \\
\hline $\mathrm{Fe}(\mathrm{mg})$ & 4,9 & 1,5 & 1,7 & 3,12 & 1,7 & 1,5 & 2 & 1,6 & 2,1 & 2,19 & 1,6 & 3,8 & 2,5 & 2,4 & 7,0 & 2,4 \\
\hline $\mathrm{Zn}(\mathrm{mg})$ & 5,0 & 1,5 & 1,7 & 2,56 & 2 & 1,7 & 2 & 1,5 & 2,4 & 2,58 & 1,8 & 2,9 & 1,9 & 1,8 & 5,2 & 1,8 \\
\hline I $(\mu \mathrm{g})$ & 50 & 15 & 16 & 37,6 & 18 & 20 & 30 & 23 & 46 & 49 & 25 & 32 & 32 & 20 & 56 & 20 \\
\hline $\mathrm{Cu}(\mu \mathrm{g})$ & 0,4 & 0,135 & 0,19 & 0,496 & 0,17 & 200 & 0,12 & 240 & 350 & 0,35 & 150 & 430 & 440 & 270 & 754 & 270 \\
\hline $\mathrm{Mn}(\mathrm{mg})$ & 0,8 & 0,2 & 0,35 & 0,4 & 0,27 & 0,32 & & 0,36 & 0,63 & 0,64 & 0,34 & 0,8 & 0,64 & 0,50 & 1,3 & 0,50 \\
\hline $\mathrm{F}(\mathrm{mg})$ & 0,3 & 0,1 & 0,15 & 0,304 & 0,11 & 0,15 & & 0,21 & 0,19 & 0,20 & 0,18 & 0,20 & 0,24 & 0,15 & 0,45 & 0,15 \\
\hline $\mathrm{Se}(\mu \mathrm{g})$ & 17 & 4,5 & 8 & 16 & 9,5 & 12 & 12 & 10 & 14 & 15,4 & 9,0 & 14 & 11 & 8,6 & 26 & 8,6 \\
\hline $\mathrm{Cr}(\mu \mathrm{g})$ & 17 & 5,3 & 7,5 & 16 & 8 & 6,5 & 13 & 15 & 13 & 13 & 4,2 & 16 & 13 & 10 & 20 & 10 \\
\hline Mo $(\mu \mathrm{g})$ & 32 & 6,0 & 13 & 19,2 & 14 & 15 & 20 & 18 & 20 & 21,5 & 7,0 & 24 & 19 & 15 & 44 & 15 \\
\hline VITAMINAS & & & & 0 & & & & & & & & & & & & \\
\hline Vitamina A ( $\mu \mathrm{g})(\mathrm{RE})$ & 226 & 61 & 138 & 200 & 120 & 165 & 170 & 170 & 260 & 288 & 116 & 240 & 205 & 123 & 419 & 123 \\
\hline Vitamina D ( $\mu \mathrm{g})$ & 5,4 & 1,5 & 1,8 & 2,48 & 1,4 & 2 & 11 & 2,2 & 2,1 & 2,08 & 2,6 & 1,8 & 1,7 & 1,1 & 6,5 & 1,5 \\
\hline Vitamina $E(m g)(a-T E)$ & 6,5 & 1,9 & 3 & 4,96 & 2,7 & 4 & 3,5 & 2,6 & 3,7 & 4,9 & 2,5 & 3,8 & 2,7 & 1,9 & 5,2 & 1,9 \\
\hline Vitamina K ( $\mu \mathrm{g})$ & 22 & 6,0 & 14 & 16,8 & 14 & 14 & 14 & 12 & 16 & 18,9 & 11 & 13 & 13 & 8,0 & 23 & 8,0 \\
\hline Tiamina (mg) & 0,8 & 0,2 & 0,23 & 0,344 & 0,15 & 0,2 & 0,25 & 0,24 & 0,45 & 0,52 & 0,19 & 0,40 & 0,37 & 0,23 & 0,58 & 0,23 \\
\hline Riboflavina (B2) (mg) & 0,8 & 0,2 & 0,22 & 0,4 & 0,22 & 0,22 & 0,38 & 0,27 & 0,50 & 0,56 & 0,20 & 0,40 & 0,4 & 0,24 & 0,73 & 0,24 \\
\hline Niacina (mg) (NE) & 8,0 & 1,7 & 2,5 & 2 & 3,2 & 3 & 3,7 & 2,7 & 3,6 & 0,70 & 2,5 & 4,3 & 3,3 & 2,7 & 4,2 & 2,7 \\
\hline Ácido Pantoténico (mg) & 1,7 & 0,5 & 0,85 & 1,28 & 0,65 & 0,88 & 1,4 & 0,86 & 1,6 & 1,5 & 0,83 & 1,3 & 1,3 & 0,80 & 2,3 & 0,80 \\
\hline Vitamina B6 (mg) & 0,6 & 0,2 & 0,35 & 0,76 & 0,24 & 0,35 & 0,38 & 0,28 & 0,53 & 0,61 & 0,27 & 0,40 & 0,42 & 0,26 & 0,73 & 0,26 \\
\hline Ácido Fólico $(\mu \mathrm{g})$ & 88 & 23 & 45 & 72 & 39 & 40 & 35 & 45 & 80 & 80,9 & 41 & 64 & 64 & 40 & 112 & 40 \\
\hline Vitamina B12 ( $\mu \mathrm{g})$ & 0,9 & 0,3 & 0,22 & 0,76 & 0,57 & 0,72 & 0,3 & 0,58 & 1,1 & 0,9 & 0,5 & 0,70 & 0,51 & 0,32 & 1,2 & 0,32 \\
\hline Biotina $(\mu \mathrm{g})$ & 17 & 6,0 & 6,3 & 15,2 & 4,7 & 7 & 9 & 7 & 12 & 10,1 & 5,0 & 9,6 & 6,4 & 6,0 & 12 & 6,0 \\
\hline Vitamina C (mg) & 58 & 15 & 15 & 29,6 & 20 & 17 & 15 & 16 & 30 & 30,7 & 17 & 24 & 16 & 15 & 44 & 15 \\
\hline \multicolumn{17}{|l|}{$\begin{array}{l}\text { DISTRIBUIÇÃO DE } \\
\text { ENERGIA }\end{array}$} \\
\hline Proteína (En \%) & 9 & 8,7 & 15 & & 25 & 18 & 23 & 15 & 24 & 24 & 24 & 16 & 25 & 16 & 20 & 16 \\
\hline Lípidos (En \%) & 41 & 40,2 & 30 & & 35 & 43 & 38 & 35 & 35 & 35 & 31 & 35 & 28 & 35 & 30 & 35 \\
\hline $\begin{array}{l}\text { Hidratos de } \\
\text { Carbono (En \%) }\end{array}$ & 50 & 49,1 & 55 & & 40 & 39 & 39 & 50 & 41 & 41 & 45 & 49 & 47 & 49 & 49 & 49 \\
\hline $\begin{array}{l}\text { OSMOLARIDADE } \\
\text { (mOsml/L) }\end{array}$ & 425 & 440 & 488 & & 480 & 520 & & 382 & & 570 & 440 & 790 & 600 & 455 & 330 & 360 \\
\hline SABORES & $\begin{array}{l}\text { Neutro e } \\
\text { Baunilha }\end{array}$ & $\begin{array}{l}\text { Baunilha, } \\
\text { Banana, } \\
\text { Chocolate }\end{array}$ & $\begin{array}{c}\text { Alperce, } \\
\text { baunilha, } \\
\text { chocolate, } \\
\text { morango }\end{array}$ & $\begin{array}{l}\text { Baunilha, } \\
\text { morango }\end{array}$ & $\begin{array}{l}\text { Baunilha, } \\
\text { morango }\end{array}$ & $\begin{array}{l}\text { Alperce, } \\
\text { baunilha }\end{array}$ & $\begin{array}{l}\text { Baunilha e } \\
\text { frutos do } \\
\text { bosque }\end{array}$ & Baunilha & $\begin{array}{l}\text { Banana, } \\
\text { baunilha, } \\
\text { café, } \\
\text { morango e } \\
\text { pêssego- } \\
\text {-manga }\end{array}$ & $\begin{array}{l}\text { Neutro, } \\
\text { frutos } \\
\text { vermelhos } \\
\text { sensação } \\
\text { refrescante } \\
\text { e gengibre } \\
\text { tropical }\end{array}$ & $\begin{array}{l}\text { Baunilha, } \\
\text { chocolate, } \\
\text { morango }\end{array}$ & $\begin{array}{c}\text { Banana, } \\
\text { baunilha, } \\
\text { café, } \\
\text { morango e } \\
\text { neutro }\end{array}$ & $\begin{array}{l}\text { Baunilha, } \\
\text { café, choco- } \\
\text { late e frutos } \\
\text { silvestres }\end{array}$ & $\begin{array}{l}\text { Baunilha, } \\
\text { banana e } \\
\text { morango }\end{array}$ & Neutro & \\
\hline VIA ADMINISTRAÇÃO & Oral & Oral & $\begin{array}{l}\text { Oral ou } \\
\text { entérica por } \\
\text { sonda }\end{array}$ & $\begin{array}{l}\text { Oral ou } \\
\text { entérica por e } \\
\text { sonda }\end{array}$ & $\begin{array}{l}\text { Oral ou } \\
\text { entérica por } \\
\text { sonda }\end{array}$ & $\begin{array}{l}\text { Oral ou } \\
\text { entérica por } \\
\text { sonda }\end{array}$ & $\begin{array}{l}\text { Oral ou } \\
\text { entérica por } \\
\text { sonda }\end{array}$ & $\begin{array}{l}\text { Entérica por } \\
\text { sonda ou } \\
\text { oral }\end{array}$ & Oral & Oral & Oral & Oral & Oral & Oral & Oral & Sonda \\
\hline
\end{tabular}

Produtos a sombreado correspondem a produtos específicos para a idade pediátrica 
Dietas poliméricas hipoenergéticas e normoenergéticas disponíveis no mercado português

\begin{tabular}{|c|c|c|c|c|c|c|c|c|c|c|c|c|c|c|c|c|c|c|c|c|}
\hline & $\begin{array}{l}\text { DIETAS } \\
\text { OLIMÉRIC, } \\
\text { RENERGÉ } \\
\end{array}$ & & & & & & DIETAS PC & OLIMÉRIC & ICAS NORI & IMOENERC & IGÉTICAS & & & & & & & & & \\
\hline $\begin{array}{l}\text { DESIGNACÃO } \\
\text { COMERCIAL }\end{array}$ & B1 & $c 1$ & C2 & C3 & C4 & C5 & C6 & C7 & C8 & c9 & C10 & c11 & $\mathrm{C}_{12}$ & $\mathrm{C13}$ & C14 & C15 & C16 & c17 & C18 & C19 \\
\hline $\begin{array}{l}\text { CARACTERISTICAS } \\
\text { NUTRICIONAIS/100ML } \\
\text { OU } 100 \mathrm{G}\end{array}$ & $100 \mathrm{ML}$ & $100 \mathrm{ML}$ & $100 \mathrm{ML}$ & $100 \mathrm{G}$ & $100 \mathrm{ML}$ & $100 \mathrm{ML}$ & 100ML & $100 \mathrm{ML}$ & $100 \mathrm{ML}$ & $100 \mathrm{ML}$ & $100 \mathrm{ML}$ & $100 \mathrm{ML}$ & $100 \mathrm{ML}$ & $100 \mathrm{ML}$ & $100 \mathrm{ML}$ & $100 \mathrm{ML}$ & $100 \mathrm{ML}$ & $100 \mathrm{ML}$ & $100 \mathrm{ML}$ & $100 \mathrm{ML}$ \\
\hline ENERGIA (kcal/ kJ) & $50 / 210$ & $100 / 4201$ & $101 / 4205$ & $500 / 2095$ & $5100 / 420$ & $100 / 420$ & $100 / 4201$ & $100 / 420$ & $100 / 420$ & $420 / 4,0$ & $103 / 430$ & $125 / 525$ & $128 / 535$ & $126 / 528$ & $104 / 435$ & $101 / 422$ & $125 / 527$ & $\begin{array}{cc}103 / \\
433,6\end{array}$ & $103 / 433$ & $3100 / 422$ \\
\hline PROTEÍNAS (g) & 2,0 & 2,5 & 2,6 & 13 & 2,5 & 3,3 & 3,8 & 3,8 & 3,8 & 1 & 4,0 & 6,3 & 6,3 & 10 & 5,5 & 3,6 & 9,4 & 3,9 & 3,9 & 3,9 \\
\hline LíPIDOS (g) & 2,0 & 4,4 & 5,4 & 27 & 4,4 & 4,2 & 3,4 & 3,4 & 3,4 & & 3,9 & 4,9 & 4,9 & 4,9 & 3,3 & 4,7 & 3,5 & 3,4 & 3,4 & 3,4 \\
\hline MCT (g) & & 0,89 & 0,61 & & & & 0 & 0 & 0 & 1,0 & & 0,7 & 0,8 & & & 1,2 & & 0,7 & & 0,7 \\
\hline AGS (g) & 0,2 & 0,29 & 2,2 & 11,4 & 0,5 & 0,5 & 0,3 & 0,3 & 0,3 & 2,2 & 1,0 & 1,3 & 1,3 & 1,3 & 1,2 & 2,6 & 0,5 & 1,2 & 1,2 & 1,2 \\
\hline AGMI (g) & 1,2 & 2,1 & 2,1 & 9,6 & 2,6 & 2,4 & 2,2 & 2,1 & 2,1 & 0,7 & 2,2 & 2,7 & 2,7 & 2,6 & 1,4 & 1,1 & 2,1 & 1,5 & & 1,5 \\
\hline AGPI (g) & 0,6 & 1,16 & 1,1 & 4,8 & 1,3 & 1,3 & 0,9 & 1 & 1 & 12,3 & 0,7 & 0,9 & 0,9 & 0,96 & 0,7 & 0,5 & 0,9 & 0,7 & & 0,7 \\
\hline $\begin{array}{l}\text { HIDRATOS DE } \\
\text { CARBONO (g) }\end{array}$ & 6,2 & 12,5 & 10,3 & 50 & 12,5 & 12,3 & 13,8 & 13,8 & 13 & 12,3 & 12,3 & 14,2 & 14,1 & 10,4 & 12,5 & 11 & 14 & 13,5 & 13,5 & 13,5 \\
\hline Lactose (g) & $<0,025$ & $\leq 0,03$ & 5,2 & 27 & $<0,025$ & $<0,025$ & $\leq 0,01$ & $\leq 0,01$ & $\leq 0,01$ & 11,4 & $<0,025$ & $<0,025$ & $<0,025$ & $<0,025$ & $<0,025$ & $<0,02$ & $<0,5$ & $<0,05$ & & $<0,05$ \\
\hline Outros (g) & 0,5 & & & & & & & & & & & & & & & & & & & \\
\hline $\begin{array}{l}\text { FIBRA SOLÚVEL/ } \\
\text { INSOLÚVEL (g) }\end{array}$ & & 0 & 0,6 & 2,8 & 0 & 0 & 0 & 0 & 1,5 & & 1,5 & $<0,1$ & 1,5 & 0,09 & 1,5 & & $0 /<0,5$ & 1,5 & 1,5 & 0 \\
\hline $\begin{array}{l}\text { MINERAIS/ } \\
\text { OLIGOELEMENTOS }\end{array}$ & & & & & & & & & & & & & & & & & & 0 & & \\
\hline $\mathrm{Na}(\mathrm{mg})$ & 50 & 54 & 37 & 185 & 60 & 80 & 75 & 75 & 133 & 150 & 100 & 111 & 100 & 116 & 100 & 35 & 80 & 80 & & 80 \\
\hline $\mathrm{K}(\mathrm{mg})$ & 75 & 100 & 95 & 499 & 110 & 138 & 125 & 125 & 155 & 125 & 150 & 168 & 168 & 218 & 150 & 122 & 155 & 135 & & 135 \\
\hline $\mathrm{Cl}$ (mg) & 63 & 82 & 62 & 275 & 95 & 110 & 85 & 115 & 153 & 80 & 125 & 80 & 80 & 96,4 & 125 & 74 & 100 & 100 & & 135 \\
\hline $\mathrm{Ca}$ (mg) & 40 & 70 & 100 & 500 & 60 & 70 & 60 & 80 & 80 & 72 & 80 & 90 & 90 & 75 & 80 & 91 & 120 & 70 & & 70 \\
\hline $\mathrm{P}(\mathrm{mg})$ & 36 & 61 & 50 & 280 & 50 & 60 & 47 & 63 & 63 & 23 & 72 & 90 & 90 & 81,9 & 72 & 61 & 100 & 60 & & 60 \\
\hline $\mathrm{Mg}(\mathrm{mg})$ & 23 & 12,5 & 9,0 & 40 & 11 & 17 & 20 & 25 & 25 & 1,6 & 23 & 28 & 28 & 22 & 23 & 20 & 23 & 17 & & 17 \\
\hline $\mathrm{Fe}(\mathrm{mg})$ & 1,6 & 1 & 1,2 & 5 & 1 & 1,3 & 2 & 1,3 & 1,3 & 1,2 & 1,6 & 2,0 & 2,0 & 2 & 1,6 & 1,1 & 1,5 & 1,1 & & 1,1 \\
\hline $\mathrm{Zn}(\mathrm{mg})$ & 1,2 & 1 & 0,8 & 4,5 & 1 & 1,1 & 1,5 & 1,2 & 1,2 & 13 & 1,2 & 1,5 & 1,5 & 1,45 & 2,0 & 0,96 & 1,3 & 1 & & 1 \\
\hline I ( $\mu \mathrm{g})$ & 13 & 10 & 18 & 77 & 10 & 12 & 30 & 13,1 & 13,3 & 180 & 13 & 17,0 & 17 & 18,9 & 13 & 10 & 17 & 13 & & 13 \\
\hline $\mathrm{Cu}(\mu \mathrm{g})$ & 180 & 100 & 70 & 3 & 8 & 10 & 0,3 & 133 & 133 & 0,33 & 180 & 230 & 230 & 0,22 & 200 & 0,1 & 0,17 & 0,17 & & 170 \\
\hline $\mathrm{Mn}(\mathrm{mg})$ & 0,33 & 0,12 & 0,02 & 0,28 & 0,2 & 0,2 & 0,4 & 0,27 & 0,27 & 0,10 & 0,33 & 0,41 & 0,41 & 0,35 & 0,38 & 0,2 & 0,27 & 0,24 & & 0,24 \\
\hline $\mathrm{F}(\mathrm{mg})$ & 0,10 & 0,08 & & & 0,1 & 0,1 & 0,2 & 0,13 & 0,13 & 5,7 & 0,10 & 0,13 & 0,13 & 0,13 & 0,1 & 2 & 0,12 & 0,13 & & 0,13 \\
\hline $\mathrm{Se}(\mu \mathrm{g})$ & 5,7 & 3 & 2,2 & 10 & 3,0 & 4,9 & 10 & 6,7 & 6,7 & 6,7 & 5,7 & 7,1 & 7,1 & 6,83 & 9,6 & 3,5 & 7,5 & 7 & & 7 \\
\hline $\operatorname{Cr}(\mu \mathrm{g})$ & 6,7 & 4 & $<8$ & 3,5 & 3,5 & 5,1 & 10 & 6,7 & 6,7 & 10 & 6,7 & 8,3 & 8,3 & 8,30 & 6,7 & 5,1 & 5,5 & 11 & & 11 \\
\hline Mo $(\mu \mathrm{g})$ & 10 & 4 & $<8$ & 7,8 & 4 & 7,0 & 15 & 10 & 10 & & 10 & 13,0 & 13 & 13,0 & 10 & 7,5 & 11 & 13 & & 13 \\
\hline VITAMINAS & & & & & & & & & & 82 & & & & & & & & 0 & & \\
\hline Vitamina A ( $\mu \mathrm{g})(\mathrm{RE})$ & 82 & 45 & 81 & 415 & 41 & 61 & 120 & 70 & 70 & 1,0 & 82 & 102 & 102 & 102 & 82 & 84 & 120 & 110 & & 110 \\
\hline Vitamina D $(\mu \mathrm{g})$ & 0,70 & 0,75 & 1,9 & 10 & 1 & 0,7 & 2 & 1,3 & 1,3 & 1,3 & 1,0 & 1,7 & 1,7 & 1,72 & 0,7 & 1 & 1,3 & 1,5 & & 1,5 \\
\hline Vitamina $\mathrm{E}(\mathrm{mg})(\mathrm{a}-\mathrm{TE})$ & 1,3 & 2 & 2,1 & 5,7 & 1,3 & 1,3 & 3 & 1,3 & 1,3 & 5,3 & 1,3 & 1,6 & 1,6 & 1,63 & 7,5 & 1,3 & 2,3 & 1,6 & & 1,6 \\
\hline Vitamina K ( $\mu \mathrm{g})$ & 5,3 & 4 & 6,7 & 30 & 4 & 4,5 & 16,7 & 6,7 & 6,7 & 0,15 & 5,3 & 6,6 & 6,6 & 6,6 & 5,3 & 5,6 & 10 & 7 & & 7 \\
\hline Tiamina (mg) & 0,15 & 0,2 & 0,15 & 0,75 & 0,2 & 0,2 & 0,23 & 0,13 & 0,13 & 0,16 & 0,15 & 0,19 & 0,2 & 0,19 & 0,15 & 0,12 & 0,2 & 0,14 & & 0,14 \\
\hline Riboflavina (B2) (mg) & 0,16 & 0,2 & 0,2 & 0,75 & 0,2 & 0,2 & 0,32 & 0,17 & 0,17 & 1,8 & 0,16 & 0,20 & 0,20 & 0,29 & 0,19 & 0,13 & 0,23 & 0,17 & & 0,17 \\
\hline Niacina (mg) (NE) & 1,8 & 1,2 & 1,2 & 9,4 & 1,1 & 1,5 & 3 & 1,6 & 1,6 & 0,53 & 1,8 & 2,3 & 2,3 & 2,31 & 1,8 & 2,1 & 1,6 & 1,6 & & 1,6 \\
\hline Ácido Pantoténico (mg) & 0,53 & 0,4 & 0,8 & 2,3 & 0,3 & 0,4 & 1,2 & 0,47 & 0,47 & 0,17 & 0,53 & 0,66 & 0,7 & 0,66 & 0,53 & 0,5 & 0,65 & 0,6 & & 0,6 \\
\hline Vitamina B6 (mg) & 0,17 & 0,11 & 0,1 & 0,3 & 0,1 & 0,2 & 0,33 & 0,16 & 0,16 & 27 & 0,17 & 0,21 & 0,2 & 0,21 & 0,2 & 0,17 & 0,25 & 0,17 & & 0,17 \\
\hline Ácido Fólico $(\mu \mathrm{g})$ & 27 & 22 & 16 & 75 & 15 & 21 & 50 & 26,7 & 26,7 & 0,21 & 27 & 33 & 33 & 33,1 & 30 & 24 & 35 & 29 & & 29 \\
\hline Vitamina B12 $(\mu \mathrm{g})$ & 0,21 & 0,2 & 0,3 & 2,1 & 0,3 & 0,2 & 0,6 & 0,27 & 0,27 & 4,0 & 0,21 & 0,26 & 0,3 & 0,52 & 0,24 & 0,32 & 0,65 & 0,38 & & 0,38 \\
\hline Biotina $(\mu \mathrm{g})$ & 4,0 & 5 & 4,0 & 12 & 4,0 & 4,0 & 7,5 & 5 & 5 & 10 & 4,0 & 5,0 & 5,0 & 5,0 & 4,0 & 3,2 & 5 & 4,6 & & 4,6 \\
\hline Vitamina C (mg) & 10 & 8 & 14 & 55 & 10 & 10 & 15 & 6,7 & 6,7 & 16 & 10 & 13 & 13 & 13,3 & 38 & 9,7 & 16 & 11 & & 1,6 \\
\hline \multicolumn{21}{|l|}{$\begin{array}{l}\text { DISTRIBUIÇÃO DE } \\
\text { ENERGIA }\end{array}$} \\
\hline Proteína (En \%) & 16 & 10 & 10,3 & 10 & 9,8 & 13 & 15 & 15 & 15 & 34 & 16 & 20 & 20 & 32 & 22 & 14,3 & 30 & 22 & & \\
\hline Lípidos (En \%) & 35 & 40 & 47,9 & 49 & 40,1 & 38 & 30 & 30 & 30 & 48 & 34 & 35 & 34 & 35 & 28 & 41,9 & 25 & 29 & & \\
\hline $\begin{array}{l}\text { Hidratos de } \\
\text { Carbono (En \%) }\end{array}$ & 49 & 50 & 40,7 & 40 & 50,1 & 49 & 55 & 55 & 52 & 255 & 47 & 45 & 44 & 33 & 47 & 43,8 & 45 & 49 & & \\
\hline $\begin{array}{l}\text { OSMOLARIDADE } \\
\text { (mOsml/L) }\end{array}$ & 140 & 220 & 305 & 400 & 200 & 225 & 330 & 220 & 285 & 15 & 250 & 275 & 285 & 275 & 315 & 290 & $390 / 450$ & 319 & & \\
\hline SABORES & & Neutro & Neutro & Neutro & Neutro & Neutro & $\begin{array}{l}\text { Baunilha, } \\
\text { Choco- } \\
\text { late }\end{array}$ & Neutro & Neutro & & & & & & & Neutro & $\begin{array}{l}\text { Alperce, } \\
\text { baunilha, } \\
\text { café, cho- } \\
\text { colate, } \\
\text { morango }\end{array}$ & - Baunilha & & \\
\hline VIA ADMINISTRAÇÃO & Sonda & Sonda & $\begin{array}{l}\text { Oral e } \\
\text { Sonda }\end{array}$ & $\begin{array}{l}\text { Oral e } \\
\text { Sonda }\end{array}$ & Sonda & Sonda & Oral & Sonda & Sonda & Sonda & Sonda & Sonda & Sonda & Sonda & Sonda & $\begin{array}{l}\text { Oral e } \\
\text { Sonda }\end{array}$ & $\begin{array}{l}\text { Oral ou } \\
\text { entérica } p \\
\text { por sonda }\end{array}$ & $\begin{array}{l}\text { Entérica } \\
\text { por sonda } \\
\text { ou oral }\end{array}$ & $\begin{array}{l}\text { Oral e } \\
\text { sonda }\end{array}$ & $\begin{array}{l}\text { Entérica } \\
\text { por sonda } \\
\text { ou oral }\end{array}$ \\
\hline
\end{tabular}

Produtos a sombreado correspondem a produtos específicos para a idade pediátrica 


\section{b) Fórmulas Oligoméricas e Monoméricas}

As fórmulas oligoméricas (semielementares) e monoméricas (elementares) são constituídas por macronutrientes que foram enzimaticamente hidrolisados, apresentando-se sob a forma prédigerida (1). Este tipo de fórmulas está indicado para indivíduos que apresentem problemas de digestão ou absorção, uma vez que os macronutrientes estão pré-digeridos, sendo facilmente absorvidos. Descrevem-se no Tabela 3 as dietas oligoméricas e monoméricas e respectivas composições em macro e micronutrientes.

\section{b.1) Fórmulas Oligoméricas}

Nas fórmulas oligoméricas, os macronutrientes apresentam-se parcialmente hidrolisados. A fração proteica apresenta-se sob a forma de dipeptídeos, tripeptídeos e aminoácidos livres. Por sua vez, os hidratos de carbono encontram-se sob a forma de dissacarídeos e maltodextrinas. No que diz respeito ao conteúdo lipídico, este é constituído sobretudo por triglicerídeos de cadeia longa (fonte de ácidos gordos essenciais $\omega-3$ e $\omega$-6) e TCM. A composição em micronutrientes corresponde às doses recomendadas, sendo fórmulas nutricionalmente completas (14).

As fórmulas oligoméricas têm uma osmolaridade inferior às fórmulas monoméricas e também são melhor absorvidas.

Este tipo de fórmulas está indicado: em doentes com problemas de digestão e absorção, insuficiência enzimática pancreática, doença inflamatória intestinal, síndrome do intestino curto, obstrução intestinal e/ou fístulas e enterite causada por radioterapia em doentes com patologia oncológica.

\section{b.2) Fórmulas Monoméricas}

A fonte de proteína das fórmulas monoméricas são aminoácidos livres o que aumenta a osmolaridade da fórmula. Relativamente aos hidratos de carbono, estas fórmulas contêm na sua composição glicose e oligossacarídeos. Os lípidos estão presentes, sob a forma de TCM e/ ou ácidos gordos essenciais. No que diz respeito à composição em micronutrientes, todos os micronutrientes essenciais se encontram presentes (14)

Foi demonstrado que a absorção de azoto a nível intestinal é superior na presença de di e tripeptídeos. Como tal, a absorção de uma fórmula oligomérica é superior à de uma fórmula monomérica, estando também associada a um menor número de episódios de diarreia osmótica. Tendo em consideração este facto, tem-se verificado uma mudança no sentido de serem utilizadas as fórmulas oligoméricas, particularmente nos episódios de malabsorção.

As dietas elementares, com aminoácidos livres, devem ser equacionadas, apenas, em algumas raras situações como na anafilaxia ou nas enteropatias graves (associadas a hipoalbuminémia e desnutrição) que não responderam às dietas semielementares.

Em Portugal, em 2005, apenas existia uma fórmula pediátrica monomérica (1). Atualmente existem três fórmulas pediátricas no mercado.

\section{Em Função da Densidade Energética}

A densidade energética varia entre $0,5 \mathrm{a} 2 \mathrm{kcal} / \mathrm{mL}$. Tendo em conta este parâmetro, as fórmulas poliméricas podem ser classificadas como:

- Dietas isoenergéticas (1 a $1,2 \mathrm{Kcal} / \mathrm{mL}$ )

- Dietas hipoenergéticas e hipoosmolares $(<1 \mathrm{kcal} / \mathrm{mL})$

- Dietas hiperenergéticas $(1,5-2 \mathrm{kcal} / \mathrm{mL})$

\section{Fórmulas Adaptadas a Determinadas Patologias}

Estão disponíveis no mercado fórmulas específicas adaptadas às diferentes situações clínicas. São dietas que, embora destinadas ao adulto e adolescente, poderão eventualmente ser utilizadas em crianças a partir dos 8-10 anos (1). Atualmente, existem fórmulas especialmente concebidas para doenças hepáticas, doenças renais, diabetes, insuficiência pulmonar, insuficiência cardíaca, disfunção gastrointestinal, bem como situações de stress metabólico, como traumatismos e sépsis. Na Tabela 4, descrevem-se as dietas adaptadas a determinadas patologias e respetivas composições em macro e micronutrientes.

\section{a) Fórmulas para Doentes com Patologia Hepática}

As fórmulas especializadas para insuficiência hepática e encefalopatia hepática têm uma proporção maior de aminoácidos de cadeia ramificada (valina, leucina e isoleucina) e de triglicerídeos de cadeia média. A maioria das dietas é pobre em proteínas e eletrólitos, e ligeiramente hipercalórica (>1 kcal/mL), devido a restrições de fluidos. Essas dietas devem ser reservadas para pacientes com função intestinal normal, exibindo encefalopatia hepática e não respondendo a fórmulas padronizadas (1).

\section{b) Fórmulas para Doentes com Patologia Renal}

Estas fórmulas possuem baixo teor em eletrólitos (sódio, potássio, cloro, fósforo) e alto em vitaminas hidrossolúveis, o que poderá permitir compensar as perdas intradialíticas de vitaminas (13). Como o equilíbrio hídrico e eletrólitos precisa de ser cuidadosamente monitorizado nestes pacientes (pacientes com insuficiência renal aguda são geralmente hipercatabólicos e hipermetabólicos), as dietas entéricas renais são hipercalóricas para facilitar o controlo de fluídos.

\section{c) Fórmulas para Doentes com Diabetes}

A maioria dos doentes diabéticos pode utilizar uma fórmula enteral "padrão", desde que exista uma monitorização cuidadosa da glicose no sangue e uma correta administração da medicação (antidiabéticos orais ou insulina). A maioria das dietas entéricas padrão (poliméricas) têm a seguinte composição (1):

- Proteína: $15 \%$ do valor energético total

- Lipídios: 30\% do valor energético total (1/3 ácidos gordos polinsaturados, 1/3 ácidos gordos monoinsaturados) - Hidratos de carbono: 55\% do valor energético total - Alto teor em fibra.

\section{d) Fórmulas com Ação Imunomoduladora}

Estas fórmulas especializadas pretendem ter um efeito benéfico sobre o sistema imunitário. Nutrientes específicos são frequentemente encontrados nessas fórmulas individualmente ou em combinação, nomeadamente ácidos gordos ómega-3, glutamina, arginina, vitamina A, vitamina E, selénio, zinco e nucleotídeos. Essas dietas mostraram alguns resultados promissores em pacientes críticos, nomeadamente pós-cirúrgicos e nos doentes com sépsis (15).

\section{e) Fórmulas com Ação na Disfunção Gastrointestinal (Gl)}

Os doentes com disfunção gastrointestinal, isto é, insuficiência pancreática, intestino curto, doença inflamatória do intestino, diverticulose, isquemia, etc., podem beneficiar de fórmulas hidrolizadas ou com péptidos. A função intestinal pode ser estimulada pela suplementação das fórmulas com glutamina ou fibra solúvel (fermentável) que é um precursor de ácidos gordos de cadeia curta $(13,15)$. A nutrição enteral enriquecida em Transforming Growth Factor- $\beta$ (TGF- $\beta$ ), uma citoquina anti-inflamatória, pode ser benéfica, e contribuir para a indução, e manutenção, da remissão em doentes com a doença de Crohn $(17,18)$. 
Tabela 3

Dietas semielementares e elementares disponíveis no mercado português

\begin{tabular}{|c|c|c|c|c|c|c|c|c|c|}
\hline \multirow[b]{2}{*}{$\begin{array}{l}\text { DESIGNACÃO } \\
\text { COMERCIAL }\end{array}$} & \multicolumn{6}{|c|}{ SEMIELEMENTARES } & \multicolumn{3}{|c|}{ ELEMENTARES } \\
\hline & D1 & D2 & D3 & D4 & D5 & D6 & E1 & E2 & E3 \\
\hline $\begin{array}{c}\text { CARACTERISTICAS } \\
\text { NUTRICIONAIS/100ML OU } 100 \mathrm{G}\end{array}$ & $100 \mathrm{ML}$ & $100 \mathrm{ML}$ & $100 \mathrm{ML}$ & $100 \mathrm{ML}$ & $100 \mathrm{G}$ & $100 \mathrm{ML}$ & $100 \mathrm{G}$ & $100 \mathrm{G}$ & $100 \mathrm{G}$ \\
\hline ENERGIA (kcal/ kJ) & $100 / 420$ & $100 / 420$ & $100 / 420$ & $100 / 420$ & $402 / 1700$ & $100 / 425$ & $483 / 2020$ & $475 / 1992$ & 427 \\
\hline PROTEÍNAS (g) & 2,6 & 2,8 & 4,65 & 4,5 & 14,7 & 4,0 & 13 & 13,3 & 12,5 (EP) \\
\hline $\begin{array}{l}\text { Proteínas do Leite } \\
\text { de Vaca }(\mathrm{g})\end{array}$ & & & 4,65 & 4,5 & & & & & \\
\hline LíPIDOS (g) & 5,4 & 3,9 & 2,8 & 2,8 & 6,7 & 1,7 & 24,5 & 22 & 17,5 \\
\hline AGS (g) & 3,4 & 2,2 & 0,13 & 0,15 & 4,0 & 1,0 & 8,9 & 9,4 & 6,7 \\
\hline AGMI (g) & 1,3 & 0,5 & 0,79 & 0,61 & 0,8 & 0,2 & 9,6 & 7,3 & 7,4 \\
\hline AGPI (g) & 0,7 & 1,2 & 0,55 & 0,6 & 1,9 & 0,5 & 4,8 & 4,0 & 2,4 \\
\hline AA/DHA/EPA (mg) & $15,8 / 15,7 / 3,8$ & & 0 & 0,04 & & & $81,6 / 81,6$ & & \\
\hline TCM (\%) & $50 \%$ & $46 \%$ & $12 \%$ & $13 \%$ & & 0,8 & & $35 \%$ & $35 \%$ \\
\hline $\begin{array}{l}\text { HIDRATOS DE } \\
\text { CARBONO (g) }\end{array}$ & 10,3 & 13,7 & 14,1 & 14,3 & 71 & 17,6 & 52,5 & 56 & 55 \\
\hline Lactose (g) & 0,1 & 0,1 & $\leq 0,1$ & $\leq 0,1$ & 0,5 & 0,1 & 0 & 0 & $<0,1$ \\
\hline Açúcares (g) & 2,65 & 0,83 & 5 & 1,1 & 5,9 & 1,7 & 4,7 & 5 & 23,3 \\
\hline Sacarose (g) & & & 4,25 & 0 & & & & & \\
\hline FIBRA SOLÚVEL/INSOLÚVEL (g) & 0 & 0 & 0,08 & 0,08 & & & 0 & 0 & 0 \\
\hline \multicolumn{10}{|l|}{ MINERAIS/ OLIGOELEMENTOS } \\
\hline $\mathrm{Na}(\mathrm{mg})$ & 32 & 60 & 80 & 80 & 402 & 100 & 189 & 285 & 305 \\
\hline $\mathrm{K}(\mathrm{mg})$ & 108 & 110 & 200 & 200 & 603 & 150 & 525 & 546 & 466 \\
\hline $\mathrm{Cl}(\mathrm{mg})$ & 64 & 95 & 83 & 83 & 503 & 125 & 386 & 437 & 333 \\
\hline $\mathrm{Ca}(\mathrm{mg})$ & 80 & 60 & 65 & 65 & 322 & 80 & 475 & 428 & 245 \\
\hline $\mathrm{P}(\mathrm{mg})$ & 40 & 50 & 48 & 48 & 267 & 72 & 341 & 309 & 200 \\
\hline $\mathrm{Mg}(\mathrm{mg})$ & 8,0 & 11 & 25 & 25 & 84 & 23 & 51 & 66,5 & 81,6 \\
\hline $\mathrm{Fe}(\mathrm{mg})$ & 1,0 & 1,0 & 1,3 & 1,3 & 6,4 & 1,6 & 7,3 & 5,7 & 4,2 \\
\hline $\mathrm{Zn}(\mathrm{mg})$ & 0,9 & 1,0 & 1,2 & 1,2 & 4,8 & 1,2 & 5,3 & 5,2 & 4,2 \\
\hline I ( $\mu \mathrm{g})$ & 15 & 10 & 13,3 & 13,3 & 54 & 13 & 100 & 71,3 & 33,3 \\
\hline $\mathrm{Cu}(\mathrm{mg})$ & 0,06 & 0,09 & 133 & 133 & 718 & 180 & 0,4 & 0,48 & 0,4 \\
\hline $\mathrm{Mn}(\mathrm{mg})$ & 75 & 0,2 & 0,27 & 0,27 & 1,33 & 0,33 & 200 & 0,29 & 0,6 \\
\hline $\mathrm{F}(\mathrm{mg})$ & & 1,0 & 0,13 & 0,13 & 0,4 & 0,10 & & & \\
\hline $\mathrm{Se}(\mu \mathrm{g})$ & 2,0 & 3,0 & 6,7 & 6,7 & 23 & 5,7 & 14,5 & 15,2 & 15 \\
\hline $\operatorname{Cr}(\mu \mathrm{g})$ & 4,0 & 3,5 & 6,7 & 6,7 & 27 & 6,7 & 10,6 & 7,6 & 15 \\
\hline Mo $(\mu \mathrm{g})$ & 6,0 & 4,0 & 10 & 10 & 33 & 10 & 11,3 & 21,4 & 33,3 \\
\hline \multicolumn{10}{|l|}{ VITAMINAS } \\
\hline Vitamina A ( $\mu \mathrm{g})(\mathrm{RE})$ & 81 & 41 & 70 & 70 & 329 & 82 & 406 & 214 & 330 \\
\hline Vitamina D3 ( $\mu \mathrm{g})$ & 1,7 & 1,0 & 1 & 1 & 2,8 & 0,70 & 8,8 & 6,2 & 2,5 \\
\hline Vitamina E (mg) (a-TE) & 1,2 & 1,3 & 1,3 & 1,3 & 5,0 & 1,3 & 4,8 & 6,7 & 6,1 \\
\hline Vitamina K ( $\mu \mathrm{g})$ & 6,7 & 4,0 & 6,7 & 6,7 & 21 & 5,3 & 42,5 & 19 & 25 \\
\hline Tiamina (B1) (mg) & 0,2 & 0,2 & 0,13 & 0,13 & 0,60 & 0,15 & 0,5 & 0,48 & 0,6 \\
\hline Riboflavina (B2) (mg) & 0,2 & 0,2 & 0,17 & 0,17 & 0,64 & 0,16 & 0,5 & 0,95 & 0,6 \\
\hline Niacina (B3) (mg) (NE) & 1,2 & 1,1 & 1,6 & 1,6 & 2,4 & 1,8 & 10,2 & 5,3 & 4,2 \\
\hline Ácido Pantoténico (mg) & 0,5 & 0,3 & 0,47 & 0,47 & 2,1 & 0,53 & 2,9 & 1,9 & 2,0 \\
\hline Vitamina B6 (mg) & 0,1 & 0,1 & 0,16 & 0,16 & 0,68 & 0,17 & 0,5 & 0,48 & 0,8 \\
\hline Ácido Fólico $(\mu \mathrm{g})$ & 15 & 15 & 26,7 & 26,7 & 107 & 27 & 64 & 71,3 & \\
\hline Vitamina B12 ( $\mu \mathrm{g})$ & 0,4 & 0,2 & 0,27 & 0,27 & 1,4 & 0,21 & 1,3 & 1,2 & 1,7 \\
\hline Biotina $(\mu \mathrm{g})$ & 2,3 & 4,0 & 5 & 5 & 16 & 4,0 & 19 & 14,3 & 18 \\
\hline Vitamina C (mg) & 12 & 10 & 8 & 8 & 40 & 10 & 51,6 & 47,5 & 28,3 \\
\hline Colina & 14 & 20 & 36,7 & 36,7 & 148 & 37 & & 95 & 91,6 \\
\hline \multicolumn{10}{|l|}{ DISTRIBUIÇÃO DE ENERGIA } \\
\hline Proteína (En \%) & 10,4 & 11 & 18,6 & 18 & 15 & 16 & 10,8 & 11,2 & \\
\hline Lípidos (En \%) & 48,6 & 35,1 & 25 & 25 & 15 & 15 & 43,5 & 41,6 & \\
\hline Hidratos de Carbono (En \%) & 41 & 53,9 & 56,4 & 57 & 70 & 69 & 43,5 & 47,2 & \\
\hline OSMOLARIDADE (mOsml/ L) & 295 & 295 & 410 & 300 & 470 & 455 & 340 & 580 & \\
\hline SABORES & Neutro & Neutro & Baunilha & Neutro & & & Neutro & Neutro & Laranja \\
\hline VIA ADMINISTRAÇÃO & & & Oral & Sonda & Oral ou sonda & Sonda & Oral e Sonda & Oral e Sonda & Oral e Sonda \\
\hline
\end{tabular}

Produtos a sombreado correspondem a produtos específicos para a idade pediátrica 
Tabela 4

Dietas poliméricas adaptadas a determinadas patologias disponíveis no mercado português

\begin{tabular}{|c|c|c|c|c|c|c|c|c|c|c|c|c|c|c|c|c|c|}
\hline \multirow[b]{2}{*}{$\begin{array}{l}\text { DESIGNAÇÃO } \\
\text { COMERCIAL }\end{array}$} & \multirow{2}{*}{$\begin{array}{c}\begin{array}{c}\text { FóRMULAS } \\
\text { RENAIS }\end{array} \\
\text { F1 } \\
\end{array}$} & \multicolumn{4}{|c|}{$\begin{array}{l}\text { FÓRMULAS COM } \\
\text { AÇĀO IMUNOMODULADORA }\end{array}$} & \multirow[b]{2}{*}{ H1 } & \multirow[b]{2}{*}{ H2 } & \multicolumn{4}{|c|}{$\begin{array}{l}\text { FóRMULAS PARA } \\
\text { DOENTES DIABÉTICOS }\end{array}$} & \multirow[b]{2}{*}{$\mathrm{H7}$} & \multirow[b]{2}{*}{ H8 } & \multicolumn{2}{|c|}{$\begin{array}{l}\text { FÓRMULAS } \\
\text { PARA DOENTES } \\
\text { HEPÁTICOS }\end{array}$} & \multirow{2}{*}{$\begin{array}{c}\text { FÓRMU- } \\
\text { LAS PARA } \\
\text { DISFUNÇĀO } \\
\text { GASTROIN- } \\
\text { TESTINAL } \\
\text { J1 }\end{array}$} & \multirow{2}{*}{$\begin{array}{c}\begin{array}{c}\text { FÓRMULAS } \\
\text { PARA } \\
\text { DISFAGIA }\end{array} \\
\text { K1 }\end{array}$} \\
\hline & & G1 & G2 & G3 & G4 & & & H3 & H4 & H5 & H6 & & & 11 & 12 & & \\
\hline $\begin{array}{l}\text { CARACTERÍSTICAS } \\
\text { NUTRICIONAIS/100ML } \\
\text { OU } 100 \mathrm{G}\end{array}$ & $100 \mathrm{ML}$ & $100 \mathrm{ML}$ & $100 \mathrm{ML}$ & $100 \mathrm{ML}$ & $100 \mathrm{ML}$ & $100 \mathrm{ML}$ & $100 \mathrm{G}$ & $100 \mathrm{ML}$ & $100 \mathrm{ML}$ & $100 \mathrm{ML}$ & $100 \mathrm{ML}$ & $100 \mathrm{ML}$ & $100 \mathrm{ML}$ & $100 \mathrm{ML}$ & $100 \mathrm{ML}$ & $100 \mathrm{ML}$ & $100 \mathrm{ML}$ \\
\hline ENERGIA (kcal/ kJ) & $200 / 840$ & $150 / 630$ & $50 / 210$ & $100 / 420$ & $101 / 427$ & $150 / 630$ & $150 / 630$ & $105 / 440$ & $150 / 630$ & $104 / 435$ & $103 / 435$ & $100 / 422$ & $106 / 445$ & $130 / 550$ & $130 / 550$ & $110 / 463$ & $210 / 880$ \\
\hline PROTEÍNAS (g) & 3 & 10 & 8,5 & 5,5 & 5,6 & 7,5 & 7,5 & 4,5 & 7,5 & 4,9 & 4,3 & 7 & 4 & 4 & 4 & 4,1 & 8,9 \\
\hline LíPIDOS (g) & 8,9 & 6,7 & 0,2 & 3,3 & 2,8 & 7 & 7 & 5 & 7 & 3,8 & 4,2 & 2,8 & 4,1 & 4,7 & 4,7 & 3,5 & 7,6 \\
\hline MCT (g) & 2,2 & 1,6 & & 1,9 & 0,61 & 1,2 & 1,2 & 0 & 1,2 & & & & 0,55 & 1,7 & 1,7 & 0,72 & \\
\hline AGS (g) & 0,5 & 1,2 & & 0,1 & 1,6 & 0,5 & 0,4 & 0,46 & 0,62 & 0,5 & 0,5 & 0,57 & 0,6 & 0,3 & 0,3 & 1,3 & 0,7 \\
\hline AGMI (g) & 4,7 & 1,6 & & 0,2 & 0,59 & 3,8 & 4,1 & 3,67 & 3,68 & & 3 & 1,7 & 3 & 1,4 & 1,4 & 1,5 & 4,7 \\
\hline AGPI (g) & 1,47 & 2,3 & & 1,1 & 0,58 & 1,5 & 1,3 & 0,87 & 1,5 & & 0,7 & 0,33 & 0,5 & 1,3 & 1,3 & 0,7 & 2,2 \\
\hline $\begin{array}{l}\text { HIDRATOS DE } \\
\text { CARBONO (g) }\end{array}$ & 26,4 & 11,6 & 3,75 & 12 & 13,4 & 13,1 & 13,1 & 9,25 & 13,1 & 11,7 & 11,3 & 10,8 & 12,8 & 17,4 & 17,4 & 14,5 & 25 \\
\hline Lactose (g) & $\leq 0,4$ & $\leq 0,5$ & $\leq 0,01$ & $\leq 0,01$ & $<0,05$ & 0,37 & $\leq 0,5$ & $\leq 0,1$ & $\leq 0,48$ & 3,6 & $<0,006$ & $<0,5$ & & $\leq 0,001$ & $\leq 0,01$ & & 0,2 \\
\hline $\begin{array}{l}\text { FIBRA SOLÚVEL/ } \\
\text { INSOLÚVEL (g) }\end{array}$ & 1,2 & 1,5 & 0 & 0 & 0 & 2 & 2 & 2,4 & 2,3 & 2 & 1,5 & 2 & 2 & 1 & 1 & 2,1 & 3,3 \\
\hline \multicolumn{18}{|l|}{$\begin{array}{l}\text { MINERAIS/ } \\
\text { OLIGOELEMENTOS }\end{array}$} \\
\hline $\mathrm{Na}(\mathrm{mg})$ & 68 & 47,5 & 92 & 138 & 0,27 & $65 / 70$ & 0,18 & 85 & 55 & 55 & 100 & 75 & 70 & 75 & 75 & 70 & 90 \\
\hline $\mathrm{K}(\mathrm{mg})$ & 100 & 128 & 52 & 207 & 134 & 130 & 130 & 143 & 180 & 100 & 150 & 110 & 122 & 120 & 120 & 155 & 250 \\
\hline $\mathrm{Cl}(\mathrm{mg})$ & 62 & 50 & & 141 & 120 & 55 & 50 & 124 & 85 & 45 & 125 & 80 & 70 & 71,5 & 71,5 & 100 & 95 \\
\hline $\mathrm{Ca}(\mathrm{mg})$ & 84 & 203 & & 80 & 80 & 150 & 150 & 88 & 155 & 53 & 80 & 135 & 95 & 80 & 80 & 100 & 155 \\
\hline $\mathrm{P}(\mathrm{mg})$ & 55 & 120 & & 60 & 0 & 95 & 95 & 59 & 110 & 47 & 72 & 80 & 122 & 53 & 53 & 65 & 145 \\
\hline $\mathrm{Mg}(\mathrm{mg})$ & 20 & 26 & & 25 & 23 & 15 & 15 & 31 & 30 & 23 & 23 & 17 & 17 & 27 & 27 & 14 & 32 \\
\hline $\mathrm{Fe}(\mathrm{mg})$ & 2 & 2,5 & & 1,33 & 1,2 & 2 & 2,4 & 1,48 & 2 & 1,6 & 1,6 & 1,3 & 1,1 & 1,33 & 1,33 & 1,2 & 3 \\
\hline $\mathrm{Zn}(\mathrm{mg})$ & 1,8 & 2 & 4 & 1,2 & 1,5 & 1,5 & 1,8 & 1,33 & 1,8 & 1,2 & 1,2 & 1 & 1,3 & 1,2 & 1,2 & 1,1 & 2,5 \\
\hline $\mathrm{I}(\mu \mathrm{g})$ & 20 & 37,5 & & 13,3 & 15 & 30 & 36 & 14,8 & 20 & 13 & 13 & 10 & 17 & 13,3 & 13,3 & 17 & 32 \\
\hline $\mathrm{Cu}(\mu \mathrm{g})$ & 200 & 375 & & 130 & 0,17 & 300 & 360 & 148 & 200 & 180 & 180 & 0,1 & 0,17 & 130 & 130 & 170 & 0,43 \\
\hline $\mathrm{Mn}(\mathrm{mg})$ & 0,4 & 0,5 & & 0,27 & 0,2 & 0,4 & 0,48 & 0,3 & 0,4 & 0,33 & 0,33 & 0,2 & 0,23 & 0,27 & 0,27 & 0,26 & 0,41 \\
\hline $\mathrm{F}(\mathrm{mg})$ & 0,3 & 0,25 & & 0,13 & 0,17 & 0,2 & 0,24 & 0,15 & 0,2 & 0,10 & 0,10 & 0,06 & 0,11 & 0,13 & 0,13 & 0,11 & 0,25 \\
\hline $\mathrm{Se}(\mu \mathrm{g})$ & 10 & 13,5 & 60 & 6,7 & 4,7 & 10 & 12 & 7,4 & 10 & 7,5 & 7,5 & 5 & 7,2 & 6,67 & 6,67 & 7 & 14 \\
\hline $\operatorname{Cr}(\mu \mathrm{g})$ & 3 & 12,5 & & 6,7 & 10 & 10 & 12 & 29,6 & 20 & 12 & 12 & 8 & 9,5 & 6,67 & 6,67 & 11 & 16 \\
\hline Mo $(\mu \mathrm{g})$ & 7 & 18,8 & & 10 & 0 & 15 & 18 & 11,1 & 15 & 10 & 10 & 7 & 12 & 150 & 150 & 13 & 24 \\
\hline \multicolumn{18}{|l|}{ VITAMINAS } \\
\hline Vitamina A ( $\mu \mathrm{g})(\mathrm{RE})$ & 48 & 150 & & 70 & 100 & 120 & 144 & 0,078 & 0,11 & 82 & 82 & 75 & 125 & 70 & 70 & 125 & 206 \\
\hline Vitamina D $(\mu \mathrm{g})$ & 1 & 2,5 & & 1 & 0,67 & 2 & 2,4 & 1,1 & 2 & 1,2 & 0,70 & 1 & 1,6 & 1 & 1 & 1,7 & 1,5 \\
\hline Vitamina E (mg) (a-TE) & 3 & 3,75 & 100 & 1,33 & 3 & 3 & 3,6 & 7,4 & 2,3 & 2,5 & 2,5 & 1,5 & 2,1 & 1,33 & 1,33 & 1,7 & 2,6 \\
\hline Vitamina K $(\mu \mathrm{g})$ & 10,5 & 21 & & 6,7 & 6,7 & 16,7 & 20 & 7,4 & 10 & 5,3 & 5,3 & 5 & 8 & 6,67 & 6,67 & 7,8 & 11 \\
\hline Tiamina (mg) & 0,26 & 0,3 & & 0,13 & 0,12 & 0,21 & 0,3 & 0,15 & 0,2 & 0,40 & 0,15 & 0,13 & 0,18 & 0,13 & 0,13 & 0,16 & 0,32 \\
\hline Riboflavina (B2) (mg) & 0,3 & 0,4 & & 0,17 & 0,17 & 0,32 & 0,4 & 0,19 & 0,26 & 0,20 & 0,16 & 0,15 & 0,18 & 0,17 & 0,17 & 0,19 & 0,34 \\
\hline Niacina (mg) (NE) & 3,4 & 3,75 & & 1,6 & $0,5(1,6)$ & 3 & 3,6 & 1,8 & 2,4 & 1,8 & 1,8 & 0,7 & 1 & 1,6 & 1,6 & 1,2 & 1,7 \\
\hline Ácido Pantoténico (mg) & 1,2 & 1,5 & & 0,47 & 0,8 & 1,2 & 1,4 & 0,52 & 0,7 & 0,80 & 0,53 & 0,6 & 0,63 & 0,47 & 0,47 & 0,7 & 1,1 \\
\hline Vitamina B6 (mg) & 0,67 & 0,43 & & 0,16 & 0,15 & 0,33 & 0,4 & 0,18 & 0,24 & 0,30 & 0,17 & 0,2 & 0,19 & 0,16 & 0,16 & 0,21 & 0,36 \\
\hline Ácido Fólico $(\mu \mathrm{g})$ & 100 & 62,5 & & 26,7 & 20 & 50 & 60 & 29,6 & 40 & 38 & 38 & 20 & 30 & 27 & 27 & 32 & 55 \\
\hline Vitamina B12 ( $\mu \mathrm{g})$ & 0,6 & 0,75 & & 0,27 & 0,4 & 0,6 & 0,7 & 0,3 & 0,4 & 0,65 & 0,50 & 0,35 & 0,5 & 0,27 & 0,27 & 0,4 & 0,42 \\
\hline Biotina $(\mu \mathrm{g})$ & 12 & 9,4 & & 5 & 7 & 7,5 & 9 & 5,6 & 7,5 & 6,5 & 4,0 & 3,5 & 5 & 5 & 5 & 5,5 & 8,2 \\
\hline Vitamina C (mg) & 10 & 18,8 & 300 & 6,67 & 6,7 & 15 & 18 & 18,5 & 12 & 15 & 15 & 7 & 11 & 6,67 & 6,67 & 11 & 21 \\
\hline \multicolumn{18}{|l|}{$\begin{array}{l}\text { DISTRIBUIÇÃO DE } \\
\text { ENERGIA }\end{array}$} \\
\hline Proteína (En \%) & 6 & 27 & 68 & 22 & 22 & 20 & 20 & 17 & 20 & 19 & 17 & 27 & 18 & 12 & 12 & 15 & 17 \\
\hline Lípidos (En \%) & 40 & 40 & 2 & 30 & 25 & 42 & 42 & 43 & 42 & 33 & 37 & 24 & 35 & 33 & 33 & 28 & 32,3 \\
\hline $\begin{array}{l}\text { Hidratos de } \\
\text { Carbono (En \%) }\end{array}$ & 53 & 31 & 30 & 48 & 53 & 35 & 35 & 35 & 35 & 45 & 43 & 45 & 43 & 53,5 & 53,5 & 53 & 47,6 \\
\hline $\begin{array}{l}\text { OSMOLARIDADE } \\
\text { (mOsml/L) }\end{array}$ & 500 & 435 & 490 & 270 & 298 & $350 / 390$ & $\mathrm{n}, \mathrm{a}$ & 270 & 450 & 365 & 300 & 218 & 187 & 360 & 330 & 290 & 510 \\
\hline SABORES & Baunilha & $\begin{array}{l}\text { Cappucci- } \\
\text { no, Frutos } \\
\text { Tropicais }\end{array}$ & Neutro & Neutro & Neutro & $\begin{array}{l}\text { Baunilha, } \\
\text { Cappuc- } \\
\text { cino }\end{array}$ & $\begin{array}{l}\text { Baunilha, } \\
\text { Morango } \\
\text { Silvestre }\end{array}$ & Neutro & Neutro & $\begin{array}{l}\text { Baunilha, } \\
\text { cappucino } \\
\text { e morango }\end{array}$ & & $\begin{array}{l}\text { Baunilha, } \\
\text { café, } \\
\text { morango }\end{array}$ & Frutas & $\begin{array}{l}\text { Cappucci- } \\
\text { no, Frutos } \\
\text { Tropicais }\end{array}$ & Neutro & Baunilha & $\begin{array}{l}\text { Baunilha e } \\
\text { morango }\end{array}$ \\
\hline VIA ADMINISTRAÇÃO & $\begin{array}{l}\text { Oral e } \\
\text { sonda }\end{array}$ & Oral & Sonda & Sonda & $\begin{array}{l}\text { Entérica } \\
\text { por sonda } \\
\text { ou oral }\end{array}$ & Oral & Oral & Sonda & Sonda & Oral & Sonda & $\begin{array}{l}\text { Oral ou } \\
\text { entérica } \\
\text { por sonda }\end{array}$ & $\begin{array}{l}\text { Entérica } \\
\text { por sonda } \\
\text { ou oral }\end{array}$ & Oral & Sonda & $\begin{array}{l}\text { Entérica por } \\
\text { sonda ou oral }\end{array}$ & Oral \\
\hline
\end{tabular}




\section{f) Fórmulas para Doentes com Patologia Pulmonar}

A deterioração do estado nutricional em doentes graves com insuficiência respiratória está associada à redução da massa muscular respiratória, fraqueza muscular e problemas com o desmame do suporte ventilatório. Pacientes com insuficiência pulmonar retêm $\mathrm{CO}_{2}$ e experimentam depleção de $\mathrm{O}_{2}$. A terapia nutricional, particularmente fórmulas ricas em hidratos de carbono, pode agravar uma insuficiência respiratória devido ao aumento da produção de $\mathrm{CO}_{2}$. Reduzir/ suspender a alimentação ou mudar para uma fórmula com maior proporção de gordura versus hidrato de carbono poderá ser uma atitude a seguir (1).

\section{Fórmulas Modulares}

Os produtos de nutrição entérica podem ser suplementados com fórmulas modulares, fornecedoras de apenas um macronutriente (hidratos de carbono, lípidos ou proteínas), proporcionando flexibilidade e versatilidade à nutrição enteral básica. Na Tabela 5, encontram-se as dietas modulares, bem como a sua composição nutricional em macro e micronutrientes.

As fórmulas modulares de hidratos de carbono são úteis para aumentar a densidade calórica, sem alterar a palatabilidade. A dextrinomaltose em pó (polímeros de glicose) fornece $4 \mathrm{kcal}$ por $1 \mathrm{~g}$ e é geralmente bem aceite pelos pacientes (1).

As fórmulas modulares proteicas poderão ser utilizadas em alguns tipos de doentes com necessidades proteicas aumentadas. As fontes mais utilizadas incluem caseína, lactalbumina, albumina do ovo, soro de leite, proteína de soja, entre outras.
As fórmulas modulares lipídicas são constituídas exclusivamente por triglicerídeos de cadeia media (TCM). Os lípidos têm alto valor energético e baixa osmolaridade e os TCM são facilmente absorvidos e metabolizados. As presentes fórmulas de TCM não contém ácidos gordos essenciais (1).

\section{CONCLUSÕES}

A nutrição entérica, na forma de dietas ou suplementos orais ou por sonda, é classificada pela legislação vigente por "alimentos para fins medicinais específicos" através do Regulamento EU nº 609/2013 e do Regulamento delegado EU 2016/128, sendo definida como produtos alimentares completos (com fórmula dietética padrão ou adaptada a doenças) ou incompletos. Estas fórmulas devem ser consumidas sob supervisão médica e devem conter a menção da patologia a que se destinam.

No artigo publicado em 2005 não existiam no mercado português fórmulas semielementares para crianças. Atualmente, existem duas fórmulas oligoméricas, uma indicada para crianças desde o nascimento até os 18 meses de idade, e outra indicada para crianças entre um e os seis anos de idade. Verificou-se também o aumento da oferta das diferentes categorias de fórmulas, nomeadamente nas fórmulas elementares (monoméricas) com um aumento de um para três produtos disponíveis.

Constata-se que nos últimos 15 anos houve uma notável evolução do número de dietas enterais comerciais, tendo por base a crescente evidência científica dos benefícios para a criança doente resultantes da intervenção com a nutrição enteral.

\section{Tabela 5}

Dietas modulares disponíveis no mercado português

\begin{tabular}{|c|c|c|c|c|c|c|c|c|c|c|c|c|}
\hline \multirow[b]{2}{*}{$\begin{array}{l}\text { DESIGNACCÃO } \\
\text { COMERCIAL }\end{array}$} & \multicolumn{4}{|c|}{ PROTEINAS } & \multicolumn{3}{|c|}{ LípIDos } & \multicolumn{3}{|c|}{ HIDRATOS DE CARBONO } & \multicolumn{2}{|c|}{ FIBRA } \\
\hline & L1 & L2 & L3 & L4 & M1 & M2 & M3 & N1 & N2 & N3 & 01 & $\mathrm{O} 2$ \\
\hline $\begin{array}{c}\text { CARACTERISTICAS } \\
\text { NUTRICIONAIS/100ML OU 100G }\end{array}$ & $100 \mathrm{~g}$ & $100 \mathrm{~g}$ & $100 \mathrm{~g}$ & $100 \mathrm{~g}$ & $100 \mathrm{ml}$ & & $100 \mathrm{ml}$ & & & $100 \mathrm{~g}$ & $100 \mathrm{~g}$ & $100 \mathrm{~g}$ \\
\hline ENERGIA (kcal/ kJ) & $360 / 1500$ & $368 / 1560$ & $371 / 1576$ & $286 / 1214$ & $500 / 2100$ & $855 / 3515$ & $900 / 3700$ & $384 / 1630$ & $381 / 1618$ & $380 / 1588$ & $220 /$ & $202 / 816$ \\
\hline PROTEÍNAS (g) & 87 & 87,2 & 90 & 71,4 & 0 & & 0 & & 0,2 & 0 & 3,4 & $<1,5$ \\
\hline $\begin{array}{l}\text { Proteínas do Leite } \\
\text { de Vaca (g) }\end{array}$ & 1 & & 1 & 0 & 53,8 & 95 & 100 & & 0 & 0 & 0,3 & 0 \\
\hline LíPIDOS (g) & 0 & & & & 13,9 & & & & & & & 0 \\
\hline MCT (g) & $<0,07$ & 1,2 & & & 2,8 & 95 & 100 & & & 0 & & 0 \\
\hline AGS (g) & $<0,155$ & & & & 24,6 & & & & & & & 0 \\
\hline AGMI (g) & $<0,075$ & & & & 12,5 & & & & & & & 0 \\
\hline AGPI (g) & $\leq 1$ & 1,2 & 0,5 & 0 & 4 & & 0 & 96 & 95 & 95 & 13,1 & 6 \\
\hline $\begin{array}{l}\text { HIDRATOS DE } \\
\text { CARBONO (g) }\end{array}$ & $\leq 1$ & 1,2 & & & 4 & & & 6,0 & 8 & 0 & & 6 \\
\hline Açúcares (g) & 0 & & & & 0,4 & & & & 0 & & 75,6 & 86 \\
\hline \multicolumn{13}{|l|}{ FIBRA SOLÚVEL/INSOLÚVEL (g) } \\
\hline MINERAIS/ OLIGOELEMENTOS & 550 & 110 & $<40$ & & 0 & & & 2 & $<20$ & & & $<200$ \\
\hline $\mathrm{Na}(\mathrm{mg})$ & 1200 & 140 & $<40$ & & 0 & & & & & & & \\
\hline $\mathrm{K}(\mathrm{mg})$ & 0 & 80 & & & 0 & & & & & & & \\
\hline $\mathrm{Cl}(\mathrm{mg})$ & 60 & 1350 & 1400 & & 0 & & & & & & & \\
\hline $\mathrm{Ca}(\mathrm{mg})$ & 240 & 700 & 740 & & 0 & & & & & & & \\
\hline$P(\mathrm{mg})$ & 0 & $\leq 20$ & & & 0 & & & & & & & \\
\hline $\mathrm{Mg}(\mathrm{mg})$ & 0 & & & & 0 & & & & & & & \\
\hline \multicolumn{13}{|l|}{$\mathrm{Fe}(\mathrm{mg})$} \\
\hline DISTRIBUIÇÃO DE ENERGIA & 97 & 95 & & & 0 & & & & 0 & & & \\
\hline Proteína (En \%) & 2 & 4 & & & 96,8 & 100 & & & 100 & & & \\
\hline Lípidos (En \%) & 1 & 1 & & & 3,2 & & & 100 & 0 & & & \\
\hline Hidratos de Carbono (En \%) & $\mathrm{n}, \mathrm{a}$ & 25 & & & & & & 97 & 95 & & & \\
\hline OSMOLARIDADE (mOsml/ L) & $\mathrm{n}, \mathrm{a}$ & & 3,71 & & 5 & & & & 1,15 & & & \\
\hline SABORES & Neutro & Neutro & Neutro & Neutro & & & Neutro & Neutro & Neutro & Neutro & & Neutro \\
\hline VIA ADMINISTRAÇÃO & Oral & Oral & Oral & Oral & Oral & Oral & Oral & Oral & Oral & Oral ou sonda & & \\
\hline
\end{tabular}




\section{AGRADECIMENTOS}

Os autores agradecem a informação detalhada relativamente à composição qualitativa e quantitativa dos produtos de nutrição entérica fornecida pelas empresas: Dieticare ${ }^{\circledR}$, Fresenius kabi ${ }^{\circledR}$, Nestlé $^{\circledR}$, Nutricia ${ }^{\circledR}$.

\section{REFERÊNCIAS BIBLIOGRÁFICAS}

1. Guerra P, Vasconcelos C, Silva D, Guerra A. Dietas Entéricas Utilizadas em Idade Pediátrica: uma Visão Atualizada na Realidade em Portugal. Acta Pediátr. Port.;2005: No1, 36: 51-60

2. Commission Directive 1999/21/EC of 25 March 1999 on dietary foods for special medical purposes. https://eurlex.europa.eu/legalcontent/EN/ALL/?uri=CELEX\%3A31999L0021.

3. Regulation (EU) No 609/2013 of the European Parliament and of the Council of 12 June 2013 on food intended for infants and young children, food for special medical purposes, and total diet replacement for weight control and repealing Council Directive 92/52/EEC, Commission Directives 96/8/EC, 1999/21/EC, 2006/125/EC and 2006/141/EC, Directive 2009/39/EC of the European Parliament and of the Council and Commission Regulations (EC) No 41/2009 and (EC) No 953/2009. https://eur-lex. europa.eu/legal-content/EN/TXT/?uri=CELEX\%3A32013R0609\&qid=1610565226496. 4. Commission Delegated Regulation (EU) 2016/128 of 25 September 2015 supplementing Regulation (EU) No 609/2013 of the European Parliament and of the Council as regards the specific compositional and information requirements for food for special medical purposes. https://eur-lex.europa.eu/legal-content/EN/ALL/?uri=CELEX\%3A32016R0128.

5. Directive 2009/39/EC of the European Parliament and of the Council of 6 May 2009 on foodstuffs intended for particularnutritional uses. https://eur-lex.europa.eu/ legal-content/en/ALL/?uri=CELEX\%3A32009L0039.

6. Food and Nutrition Board, Institute of Medicine. Dietary Reference Intakes: Applications in Dietary Planning. Washington, DC: National Academies Press, 2003.

7. Food and Nutrition Board, Institute of Medicine. Dietary Reference Intakes for Calcium, phosphorus, magnesium vitamin D, and fluoride. Washington, DC: National Academies Press, 2003.

8. Food and Nutrition Board, Institute of Medicine. Dietary Reference Intakes for thiamine, riboflavine, vitamin B12, panthotenic acid, biotine and choline. Washington, DC: National Academies Press, 1998.

9. Food and Nutrition Board, Institute of Medicine. Dietary Reference Intakes for vitamin C, vitamin E, selenium and carotenoides. Washington, DC: National Academies Press, 2000. 10. Food and Nutrition Board, Institute of Medicine. Dietary Reference Intakes for vitamin $\mathrm{A}$, vitamin $\mathrm{K}$, arsenic, boron, chromium, nickel, silicon, vanadium and zinc. Washington, DC: National Academies Press, 2001.

11. Food and Nutrition Board, Institute of Medicine. Dietary Reference Intakes for energy, carbohydrates, fiber, fat, fatty acids, cholesterol, proteins and aminoacids. Washington, DC: National Academies Press, 2006.

12. Food and Nutrition Board, Institute of Medicine. Dietary Reference Intakes for Calcium and vitamin D. Washington, DC: National Academies Press, 2011.

13. ESPGHAN Committee on Nutrition: Braegger C, Decsi T, Amil Dias J, Hartman C, Kolacek S, Koletzko B, Koletzko S, Mihatsch W, Moreno L, et al. Practical Approach to Paediatric Enteral Nutrition: A Comment by the ESPGHAN Committee on Nutrition. Journal of Pediatric Gastroenterology and Nutrition. 2010; 51: 110-122.

14. Zádak Z, Kent-Smith L. Basics in clinical nutrition: Commercially prepared formulas. The European e-Journal of Clinical Nutrition and Metabolism. 2009; 4: e212-e215. 15. Aggett P, Agostoni C, Axelsson I, et al. Non-digestible carbohydrates in the diets of infants and young children. A commentary by the ESPGHAN Committee on Nutrition. J Pediatr Gastroenterol Nutr 2003;36:329-37.

16. Kien CL. Digestible and indigestible carbohydrates. In: Koletzko B, Cooper P, Garza C, eds. Children's Nutrition-A Practical Reference Guide. Basel: Karger; 2008:42-6. 17. John K. Triantafillidis, Maria Tzouvala, Eleni Triantafyllidi. Enteral Nutrition Supplemented with Transforming Growth Factor- $\beta$, Colostrum, Probiotics, and Other Nutritional Compounds in the Treatment of Patientes with Inflamatory Bowel Disease John K. Nutrients. 2020 Apr; 12(4): 1048. Published online 2020 Apr 10. doi:10.3390/nu12041048.
18. Ferreira, T.M.R.; Albuquerque, A.; Cancela Penna, F.G.; Rosa, R.M.; Toulson, M.I.; Correia, D.; Barbosa, A.J.B.; Cunha, A.S.; Ferrari, M.L.A. Effect of oral nutrition supplements and TGF- $\beta 2$ on nutrition and inflammatory patterns in patients with active Crohn's disease. Nutr. Clin. Pract. 2019. 